\title{
Aerodynamics of Low Reynolds Number Plunging Airfoil under Gusty Environment
}

\author{
Yongsheng Lian and Wei Shyy \\ Department of Aerospace Engineering \\ University of Michigan, Ann Arbor, MI 48109
}

\begin{abstract}
$\underline{\text { Abstract }}$
It is known that plunging airfoil can produce both lift and thrust with certain combination of plunging amplitude and frequency. Motivated by our interest in micro air vehicles (MAVs), we utilize a NavierStokes equation solver to investigate the aerodynamics of a flapping airfoil. The roles of the plunging and pitching amplitude and frequency, and Strouhal number are studied. For a symmetric plunging airfoil NACA0012 at zero geometric angle of attack and chord Reynolds number of $2 \times 10^{4}$, at the same plunging frequency, it can produce either drag or thrust depending on the plunging amplitude. At the considered plunging amplitude (from $0.0125 c$ to $0.075 c$ ), the flow history has more influence than the kinematic angle of attack to determine the lift. When drag is produced, the viscous force dominates the total drag with decreasing influence as the plunging amplitude increases. For an airfoil experiencing combined plunge and pitch motion, both thrust and input power increase with the Strouhal number (within the range of 0.03 to 0.5). For the case studied, the thrust is induced by the lift, which approximately follows the curve of the kinematic angle of attack. Leading edge vortex moves downstream and interacts with the trailing edge vortex. We also study the impact of gust on stationary airfoil and flapping airfoil. Within the range of the parameters tested, for stationary airfoil the lift is in phase with the velocity but the drag is slightly out of phase. For flapping airfoil, neither lift nor drag is in phase with the velocity.
\end{abstract}

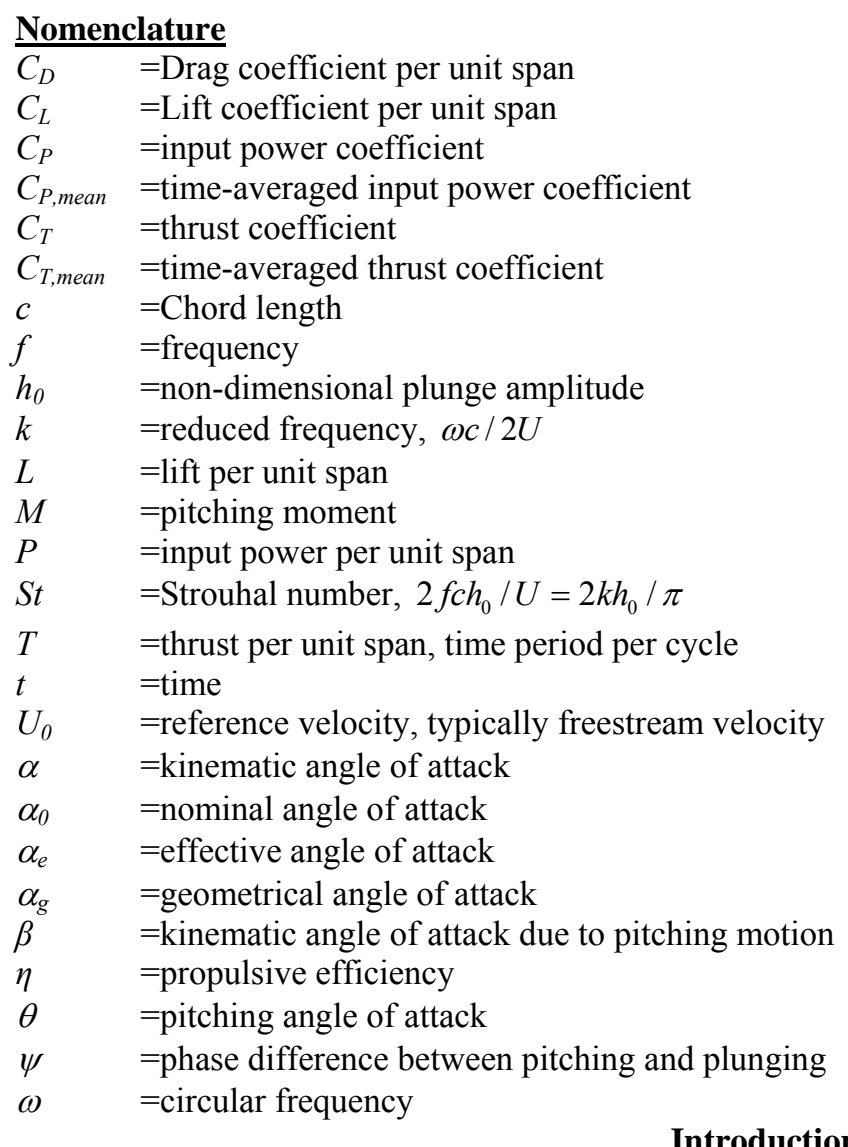

Micro Air Vehicles (MAVs), which typically has a dimension of less than $15 \mathrm{~cm}$ and flight speed around $10 \mathrm{~m} / \mathrm{s}$, operate at Reynolds numbers, based on the mean airfoil chord, of 150,000 and lower. In some parts of the flight envelop the Reynolds number can be lower than $10^{4}$. The success of MAV design requires collaborations across different disciplines including aerospace engineering, biology, and electronic 
engineering. From an aerodynamic point of view, two prominent issues needed to be understood. First, how to generate sufficient lift to keep the vehicles airborne; second, how to maintain a stable flight under gusty flight conditions. Due to their low inertia $\left(2.7 \mathrm{~g}\right.$ to $\left.\mathrm{O}\left(10^{2}\right) \mathrm{g}\right)$ and slow flight speed $(0 \sim 20 \mathrm{~m} / \mathrm{s})$ the MAVs are sensitive to the wind gust.

For lift generation, researchers have tried three types of wings, namely, fixed wing, rotary wing, and flapping wing. Fixed wings have been demonstrated in MAVs with a maximal dimension around 10-15 $\mathrm{cm}^{[1,2]}$ Figure 1(a) shows the 15-cm MAV design by Ifju et al. at the University of Florida. However, a fixed wing MAV does not possess the ability to hover in a controlled, efficient manner, which is important to many MAV missions. The rotary wing MAV, namely, a miniature helicopter, can perform controlled hovering. To date, a vehicle with $6 \mathrm{~cm}$ rotor has been successfully designed. ${ }^{[3]}$ Figure 1(b) shows a rotary wing MAV with $6 \mathrm{~cm}$ rotary diameter. Flapping flight vehicle, aimed at simultaneously integrating lift, propulsion and control, offers substantial potential for forward flight, hovering, and wind gust adaptation. ${ }^{[4,5,6]}$

There is a substantial gap between the vehicle performance goals and the design capability. In this paper, we investigate the plunging airfoil aerodynamics, especially in regard to thrust and drag characteristics. In thrust generation, the published studies have focused on the Reynolds number in the range between $6 \times 10^{3}$ to $4 \times 10^{4}{ }^{47,8,9,10]}$ It is noted that the flapping wing MAVs can employ either a fully integrated design utilizing flapping to generate lift as well as thrust, or a hybrid design utilizing flapping for thrust while relying on fixed wings for lift. Figure 1(c) is a biplane MAV design by Jones and Platzer ${ }^{[6]}$, with a $25 \mathrm{~cm}$ span. In this design, the flapping wings generate thrust while the stationary wing provides lift. The biplane design makes the vehicle less sensitive to gust and more resistant to stall. ${ }^{[6]}$ The present paper is closely related to flapping wing in Figure 1(c).

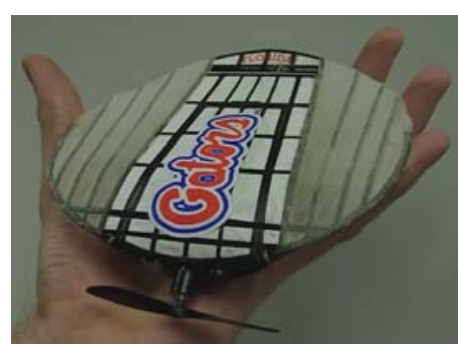

(a)

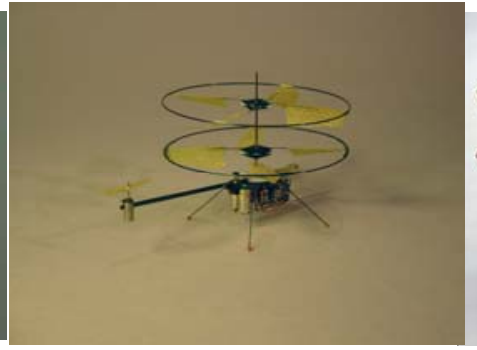

(b)

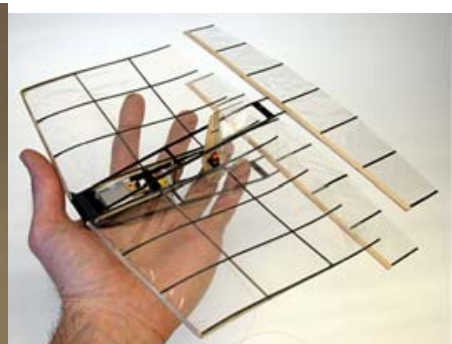

(c)

Figure 1. Three representative MAVs. (a) Fixed wing ${ }^{[2]}$; (b) rotary wing ${ }^{[3]}$; (c) flapping wing ${ }^{[6]}$.

Most of the published research on wind gust is on the dynamic loading and structural safety of manned aircraft, which means that the Reynolds number is in much higher regimes. For example, Zaide and Raveh used a Navier-Stokes equation solver to simulate the response of an airfoil to arbitrary-shaped gust ${ }^{[11]}$ with Mach number from 0.11 to 0.7. Patil and Taylor calculated the flight dynamic characteristics and gust response of highly flexible aircraft. ${ }^{[12]}$ Golubev et al. conducted parametric study of nonlinear gust-airfoil interaction for a range of gust frequencies and amplitudes in subsonic flow. ${ }^{[13]}$ Yang and Obayashi coupled a Navier-Stokes equations and dynamic equations to study the gust responses in plunging and pitching for a supersonic transport vehicle with and without the consideration of structural deformation. ${ }^{[14]}$ On the MAV front, Shyy et al. computationally and experimentally investigated the aerodynamic response of rigid and flexible airfoils to gust ${ }^{[15]}$ and reported that a flexible airfoil is more gust-tolerant than a rigid airfoil in terms of maintaining the lift to drag ratio. In their study of a low Reynolds number airfoil SD7003 in the transitional flow region, Lian and Shyy ${ }^{[16]}$ found that both the lift and drag coefficients have substantial variations under gust environment. The gust causes the formation and burst of the laminar separation bubble and ultimately affects the airfoil performance.

To support the design of MAVs, we endeavor to understand the unsteady aerodynamics inherent in the small flight vehicles. In this paper we use computational fluid dynamics (CFD) to study the fluid physics of flapping airfoils in flow regimes relevant to MAVs. Specifically, our goals are to 

amplitude in the flow structure and the force generation;

(ii) investigate the response of plunging airfoil under both steady and unsteady freestream conditions;

In the following, we first present some background in the aerodynamics of flapping airfoil. Then we introduce the numerical tools used in the work. In the numerical result part, we validate our analysis tool against some well know test cases. At last we compare the performance of flapping wing and fixed wing under gust environment.

\section{Flapping Airfoil Aerodynamics}

\section{Flapping Kinematics}

The kinematics of an airfoil experiencing a combination of harmonic plunging and pitching motion can be describes as follows:

$$
h(t) / c=h_{0} \cos \left(\omega t+\phi_{h}\right) \quad \theta(t)=\theta_{0} \cos \left(\omega t+\phi_{\theta}\right)
$$

where $h_{0}$ is the plunging amplitude non-dimensionalized by the airfoil chord $c, \theta(t)$ depicts the pitching motion of an amplitude of $\theta_{0}$ and a frequency of $\omega$. For the flapping motion $h_{0}$ is defined positive upward and pitch amplitude positive clockwise. The leading angle between pitch and plunge is defined as $\psi=\phi_{\theta}-\phi_{h}$. The kinematics can be schematically illustrated in Figure 2

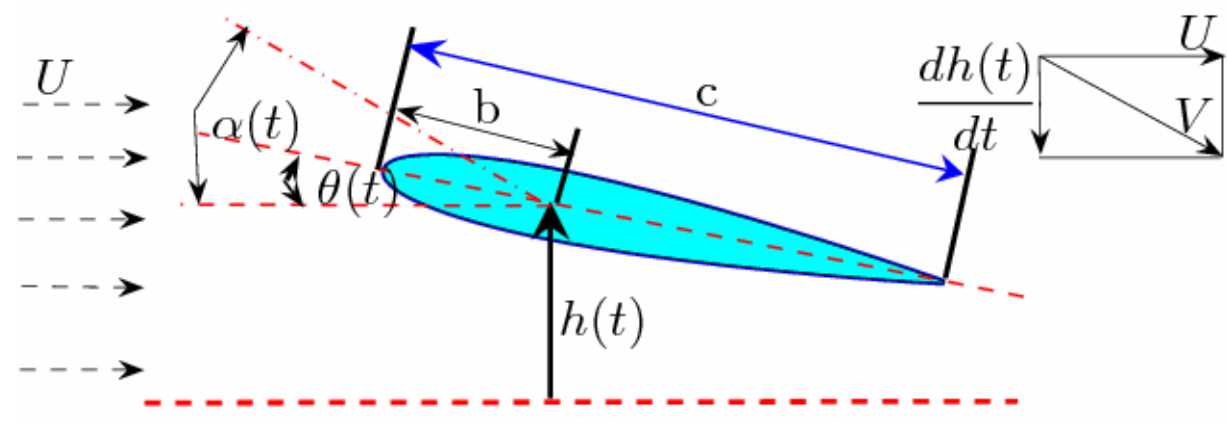

Figure 2. Schematic of flapping motion.

In the flapping wing study, two non-dimensional parameters have been employed in the literature: one is the reduced frequency defined as:

$$
k=\frac{\omega c}{2 U_{0}}
$$

another is the Strouhal number based on Anderson et al. ${ }^{[7]}$ :

$$
S t=\frac{2 f c h_{0}}{U_{0}}
$$

where $U_{0}$ is the reference velocity, typically the freestream velocity or forward flight velocity. Since there are two length scales, chord, $c$, and stroke amplitude $c h_{0}, S t$ and $k$ characterize the relative time and length scales.

\section{Different angles of attack}

To better understand the flapping wing aerodynamics, it will be useful to look at several different angles of attack attributed from different causes. The kinematic angle of attack is relative to the pivot point and is defined as the following: 


$$
\alpha(t)=\theta(t)+\beta(t)=\theta(t)+\tan ^{-1}\left(-\frac{1}{U_{0}} \frac{d h(t)}{d t}\right)=\theta_{0} \cos \left(\omega t+\phi_{\theta}\right)+\tan ^{-1}\left(\pi S t \sin \left(\omega t+\phi_{h}\right)\right)
$$

The kinematic angle of attack consists of two terms. The first term on the right side of the equation, $\theta$, is due to the pitching motion, and the second term, $\beta$, is due to the plunging motion. By definition the kinematic angle of attack is exclusively determined by the freestream velocity and the airfoil motion without considering the wake effect. More importantly, it neglects the time history of the flow. As we will discover later, flow history leads to phase lag in the force. From Eq. (4) we can readily see that the Strouhal number affects the kinematic angle of attack. Its role is demonstrated in Figure 3. At a small Strouhal number, the contribution from the plunging motion is small, and the kinematic angle of attack closely follows the shape of a sinusoidal function. As the Strouhal number increases, the kinematic angle of attack no longer follows the sinusoidal shape.

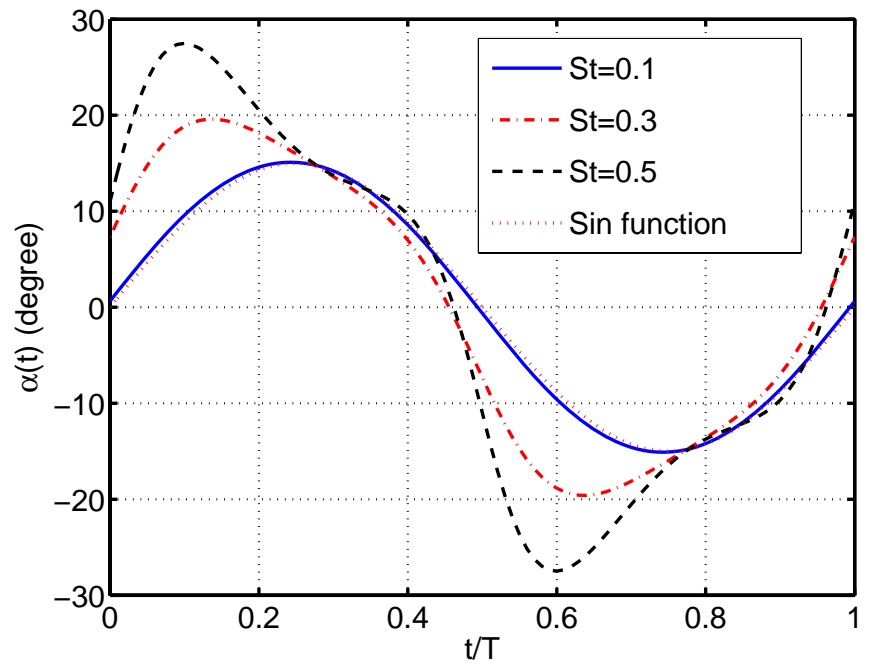

Figure 3. An instantaneous kinematic angle of attack at different Strouhal numbers. The angle of attack becomes less sinusoidal as the Strouhal number increases. $\left(h_{0}=0.75\right.$, and the nominal angle of attack, $\alpha_{0}=-\theta_{0}+\tan ^{-1}\left(2 k h_{0}\right)$, is $\left.15^{\circ}\right)$

\section{Thrust by lift}

It is known that a flapping airfoil can generate thrust at certain combinations of oscillation frequency and amplitude. This problem has been extensively investigated experimentally ${ }^{[7,17,18,19]}$ and numerically ${ }^{[9,20]}$, typically by visualizing the wake structures. Without invoking sophisticated analysis tools, we can get an intuitive idea of how the thrust is generated by looking at the local flow direction, a concept frequently used in the aerodynamics textbooks to calculate the induced drag. Consider a flapping airfoil as shown in Figure 4. The naked eye sees the airfoil at an angle of attack of $\alpha_{g}$, called geometric angle of attack and defined between the mean chord and the freestream flow direction. ${ }^{[21]}$ However, the flapping motion induces a flow velocity $U_{i}$ near the wall. Due to the flapping motion, the airfoil actually encounters the deflected local flow $U_{l}$, which is the superposition of the freestream velocity $U$ and the induced velocity $U_{i}$. During the plunging down motion, flow in the vicinity of airfoil is inclined upward with respect to the freestream, and the lift vector that remains perpendicular to the local flow direction is tilted forward. And this tilted-lift contributes to thrust. Similarly, flow is inclined downward during the plunging up motion and the lift (negative) induces thrust too. Theodorsen ${ }^{[22]}$ and Garrick, ${ }^{[23]}$ provided analytical expressions for time dependent lift, moment, and thrust on a sinusoidally plunging and pitching flat plate in a 2D potential flow. Their analysis, though has limitations, is a good starting point to study the flapping airfoil. It should be noted that Figure 4 and the associated analysis facilitate our understanding of thrust generation but to calculate the thrust requires sophisticated analysis tools with the consideration of viscous force and unsteady aerodynamics. 


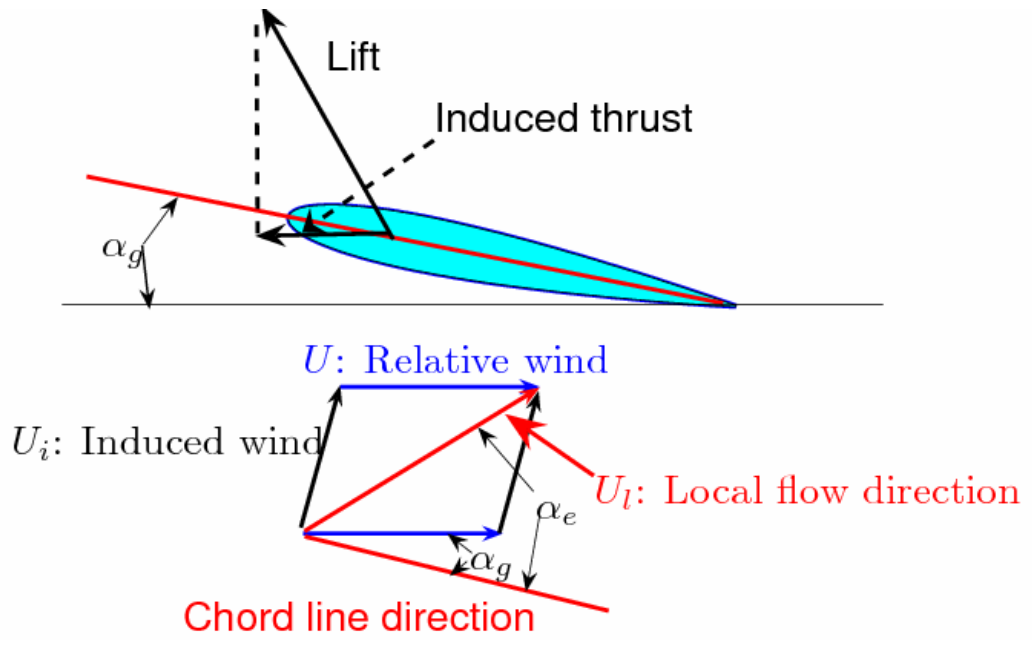

Figure 4. The origin of thrust from a flapping airfoil.

During the flapping motion, the airfoil experiences the instant force per unit span in the forward direction, force in the transverse direction, pitching moment around pivot point, and instantaneous power input, which are notated as $T(t), L(t), M(\mathrm{t})$, and $P(t)$, respectively. Among those, the input power is computed as follows:

$$
P(t)=-L(t) \frac{d h(t)}{d t}-M(t) \frac{d \theta(t)}{d t}
$$

The thrust coefficient $C_{\mathrm{T}}$ and the power input coefficient $C_{\mathrm{P}}$ are normalized as followed:

$$
C_{T}=\frac{T(t)}{\frac{1}{2} \rho U_{0}^{2} c} \quad C_{P}=\frac{P(t)}{\frac{1}{2} \rho U_{0}^{3} c}
$$

The time-averaged thrust coefficient $C_{T \text {,mean }}$ and input power coefficient $C_{p, \text { mean }}$ can be evaluated as:

$$
C_{T, \text { mean }}=\frac{1}{T} \int_{0}^{T} C_{T}(t) d t \quad C_{p, \text { mean }}=\frac{1}{T} \int_{0}^{T} C_{p}(t) d t
$$

where $T=2 \pi / \omega$. Finally, the propulsive efficiency, $\eta$, is defined as:

$$
\eta=\frac{C_{T, \text { mean }}}{C_{P, \text { mean }}}
$$

\section{Reduced frequency and Strouhal number}

There have been different opinions on the roles of these two parameters. Taylor et al. examined existing data of wingbeat frequency, stroke amplitude and flight speed of 42 species from bird, bat, and insect families. They found that $75 \%$ of the species falling in the range of $0.19<S t<0.41$ for cruising flight, with an optimum of around 0.3. ${ }^{[24]}$ Given the disparate variety of species used in the study, they implied the Strouhal number was turned for high propulsive efficiency for cruising flight or swimming. Experiments with isolated airfoils also reported high propulsive efficiencies within the interval of $\left[\begin{array}{ll}0.2 & 0.4\end{array}\right]^{[7,25,26]}$ Triantafyllou et al. stated that the Strouhal number almost solely determined the thrust efficiency. ${ }^{[25]}$ Experiments by Anderson et al. ${ }^{[7]}$ with a NACA0012 airfoil undergoing combined plunging and pitching motions at $\mathrm{Re}=4 \times 10^{4}$ also showed that the efficiency peaked at the Strouhal number range $0.25<S t<0.4$, with an high efficiency of $87 \% .{ }^{[7]}$ These evidence support the statement that the Strouhal number is the dominant parameter in using flapping wing for efficient propulsion. ${ }^{[20]}$ 
On the other hand, Bandyopadhyay et al. performed experiments by attaching a pair of flapping foils to the rear of a rigid cylinder to mimic the fish swimming. ${ }^{[27]}$ In their experiment, alternate vortex was shed from the nose of the cylinder. They found that the Strouhal number was by no means the only one to determine the thrust efficiency when there was an interaction between vortices shed from the leading-edge and flapping foils. They observed that the peak efficiency was reached below the range $0.25<S t<0.35$. In another work, Triantafyllou et al. suggested that both the flapping frequency (hence the reduce frequency) and the Strouhal number were needed to describe the flow. ${ }^{[28]}$ Experimental study by Lai and Platzer ${ }^{[19]}$ and numerical simulation by Young ${ }^{[20]}$ show that both the reduced plunging frequency $k$ and nondimensionalized amplitude $h_{0}$ should be considered separately to determine the wake structure.

From our early discussion we know that the reduced frequency characterizes the temporal property of the flapping motion while the Strouhal number considers the combined effect from temporal and spatial perspectives but ignores their individual effect. In the authors' belief, both temporal and spatial properties should be individually considered in the flapping wing study. This can be understood from the following hypothetical example. Suppose the Strouhal number is the sole parameter determining the propulsive efficiency, from Eq. (3) we know that for airfoil with chord $c$ and forward speed of $U_{0}$, as long as the $f h_{0}$ is the same, it has the same efficiency. If one combination of frequency $f$ and $h_{0}$ produces considerably high efficiency $\eta$, then we can always increase the value of $h_{0}$ and correspondingly decrease $f$ to make $f h_{0}$ constant. Under extreme situation we can make $h_{0}$ infinitely large and $f$ infinitesimally small so that the airfoil is essentially stationary. Because a stationary airfoil does not generate thrust, we know the argument that the Strouhal number is the sole parameter determining the efficiency is not true.

\section{$\underline{\text { Numerical Methods }}$}

\section{Reynolds-Averaged Navier-Stokes Solver}

The full Navier-Stokes equations in curvilinear coordinates is solved with a pressure-based algorithm, generalizing from the original Semi-Implicit Method for Pressure-Linked Equations (SIMPLE) ${ }^{[29,30]}$ The convection terms are discretized with the second-order upwind scheme and the diffusion terms are discretized with the second-order central difference scheme. The time integration is performed with a second-order implicit three-point backward scheme for better handling of accuracy and strict time step constraint imposed by the extremely fine grid resolution. The turbulence computation is realized with Wilcox's $\mathrm{k}-\omega$ turbulence model ${ }^{[31]}$ and the transitional flow simulation is carried out through a method by Lian and Shyy, ${ }^{[16]}$ which augmented a low Reynolds number $k$ - $\omega$ turbulence model ${ }^{[31]}$ with the $e^{N}$ transition method. ${ }^{[32]}$

\section{Particle tracking and Streakline integration}

Lai and Platzer visualized the wake structure of airfoil NACA0012 exerting plunging motion by releasing non-intrusive dye to the flow from the injection point. ${ }^{[19]}$ The snapshot of the wake structure is the path traveled by all particles that have passed through the injection point. It is the streakline if we ignore the mass diffusion of the dye. In the numerical analysis, the streakline can be evaluated with the following formula:

$$
\mathbf{x}\left(\mathbf{x}_{0}, t\right)=\mathbf{x}_{0}+\int_{0}^{t} \mathbf{u}(\mathbf{x}, t) d t
$$

where $\mathbf{x}_{0}$ is the injection position, $\mathbf{u}(\mathbf{x}, t)$ is the velocity vector at $\mathbf{x}$. Equation (9) is integrated with the forthorder Runge-Kutta method. The velocity vector at arbitrary position $\mathbf{x}$ is obtained with bilinear interpolation.

\section{Results and Discussion}

For demonstration purpose, we use the NACA0012 airfoil throughout this work. The readers should be aware that in real design, a slender airfoil with modest camber is more suitable for MAVs than thicker and symmetric airfoils like NACA0012. We investigate the flapping wing aerodynamics at different Reynolds 
numbers, Strouhal numbers, reduced frequencies, and freestream environments. The Reynolds number considered in this paper is $1.2 \times 10^{4}, 2.0 \times 10^{4}$ and $4.0 \times 10^{4}$, respectively. The Strouhal number covers the range from 0.03 and 0.5 , and the reduced frequency has the span from 0.2 to 3.93. Because the gust in urban environment has a low frequency around $1 \mathrm{~Hz}$, the study of flapping airfoil under gust condition involves multiple time scales. In this section, we first validate our analysis tools and then investigate the gust impact on the aerodynamics. For code validation, we will compare the vortex shedding frequency, lift and drag history, and wake structure at a variety of plunging amplitudes and frequencies. Comparisons are also made with available data from literature.

\section{Frequency validation}

Koochesfahani measured the natural shedding frequency over a stationary NACA0012 airfoil at $\operatorname{Re}=$ $1.2 \times 10^{4} \cdot{ }^{[17]}$ Even though the airfoil is streamlized, at such a low Reynolds number, it acts as a bluff body due to the separation at the trailing edge. Vortices are shed alternatively from both sides of the trailing edge. Koochesfahani reported the non-dimensionalized natural shedding frequency of 8.7. With a compressible flow solver and laminar flow simulation, Young obtained a frequency of 8.5 at a Mach number of 0.05 and 8.2 at a Mach number of $0.2 \cdot{ }^{[20]}$ At this Reynolds number, flow separates at the trailing edge without undergoing transition. Our simulation shows a frequency of 8.75. Lai and Platzer ${ }^{[19]}$ visualized the wake structure over the airfoil at the Reynolds number of $2 \times 10^{4}$, but they did not measure the shedding frequency. Based on his simulation Young reported a frequency of 9.4. ${ }^{[20]}$ Our laminar flow simulation reveals that the natural shedding frequency is 9.8. In neither case, flow experiences transition, hence our simulation is based on laminar flow assumption.

The approach of Young followed the method of Tuncer and Platzer ${ }^{[33]}$ in which the Crank-Nicolson second-order scheme was used for time discretization and the viscous flux terms were evaluated using the second-order central difference scheme and the inviscid fluxes with a third-order accurate Osher upwind scheme. However, Tuncer and Platzer used the thin-layer approximation while Young solved the full Navier-Stokes equations. Young used C-grid with $541 \times 61$ points, among them 377 points were around the airfoil surface. ${ }^{[20]}$ The first normal gridpoint was $9.2 \times 10^{-5}$ chord away from the surface. In our computation, we use O-grid with $401 \times 250$ points. The first normal gridpoint is $1.0 \times 10^{-5}$ chord away from the surface. Young investigated the effect of freestream Mach number on the numerical results. ${ }^{[20]} \mathrm{He}$ found that increasing the frequency or increasing the Mach number decreased the wavelength. As the wavelength decreased, the interaction between the pressure filed immediately close to the airfoil and the sequence of pressure wave caused by the previous airfoil motion had increasing effect on changing the force magnitude and varying the phase angles. ${ }^{[20]}$

\section{Force validation}

Lai and Platzer experimentally studied the wake structure based on a NACA0012 airfoil at the chord Reynolds number of $2 \times 10^{4}$ and various oscillation frequencies and amplitudes. ${ }^{[19]}$ Corresponding numerical simulations were performed by Young and Lai. ${ }^{\left[{ }^{[]}\right.}$To validate our code for unsteady force computation, we choose two tests. In the first case the reduced frequency, $k$, is 3.93, and the non-dimensional plunging amplitude, $h_{0}$, is 0.0125 , which give a low Strouhal number of 0.03 . Figure 5 shows the time histories of lift and drag coefficients. Same as flow over a circular cylinder, the drag varies with twice the frequency as lift. At this frequency and amplitude, the airfoil is drag-producing. Since there is no experimental measurement for force, we compare our results with the numerical results by Young. ${ }^{[34]}$ Both lift and drag coefficients show good agreement except the discrepancy in the troughs of the drag coefficient (Figure 5). With the implicit scheme, we have 100 time steps per flapping cycle while Young used explicit scheme with 16,860 time steps per flapping cycle. 

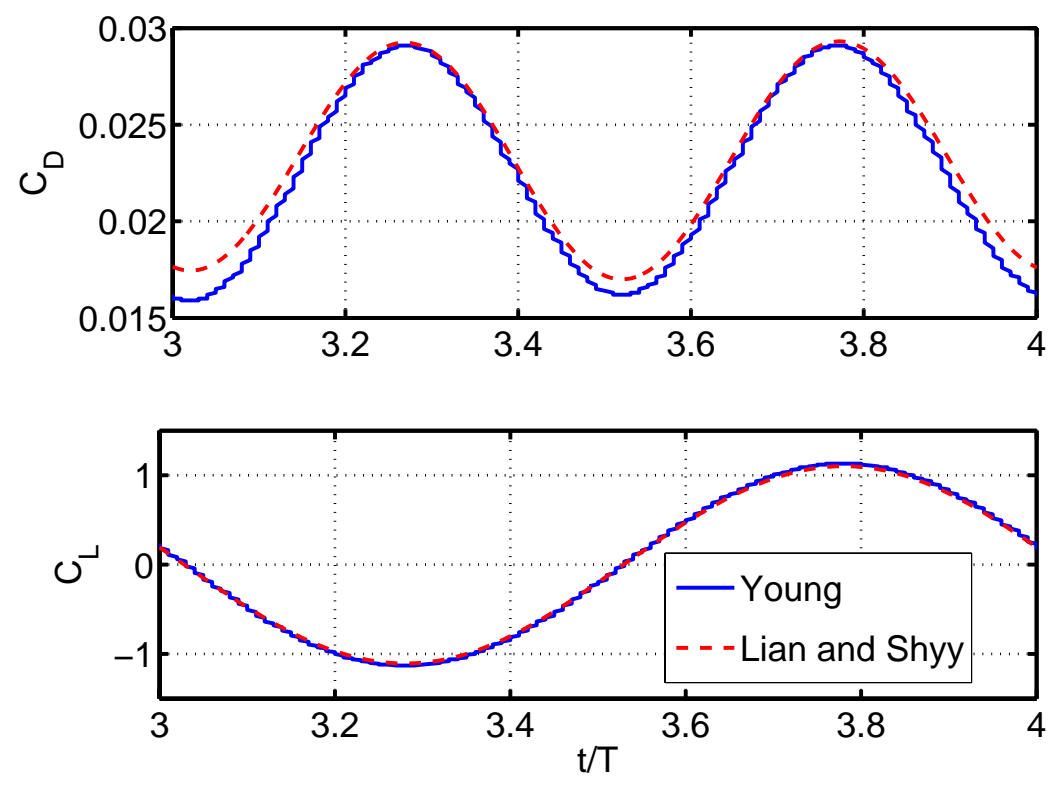

Figure 5. Drag and lift coefficient history at $k=3.93, h_{0}=0.0125$, and $S t=0.03$.

Force probe reveals that both pressure and viscous forces contribute to the total drag while the viscous force contributes almost $70 \%$ of the total drag. The time averaged viscous drag in our simulation is about $4 \%$ higher than that of Young. At this Reynolds number, we may not have adequate resolution to resolve the flow structure. With the increase of plunging amplitude, the portion of viscous force in the total drag is reduced. As shown in Figure 6, the difference between ours and that by Young actually decreases at higher oscillation amplitude. At this amplitude, the time average pressure force is thrust-generated.
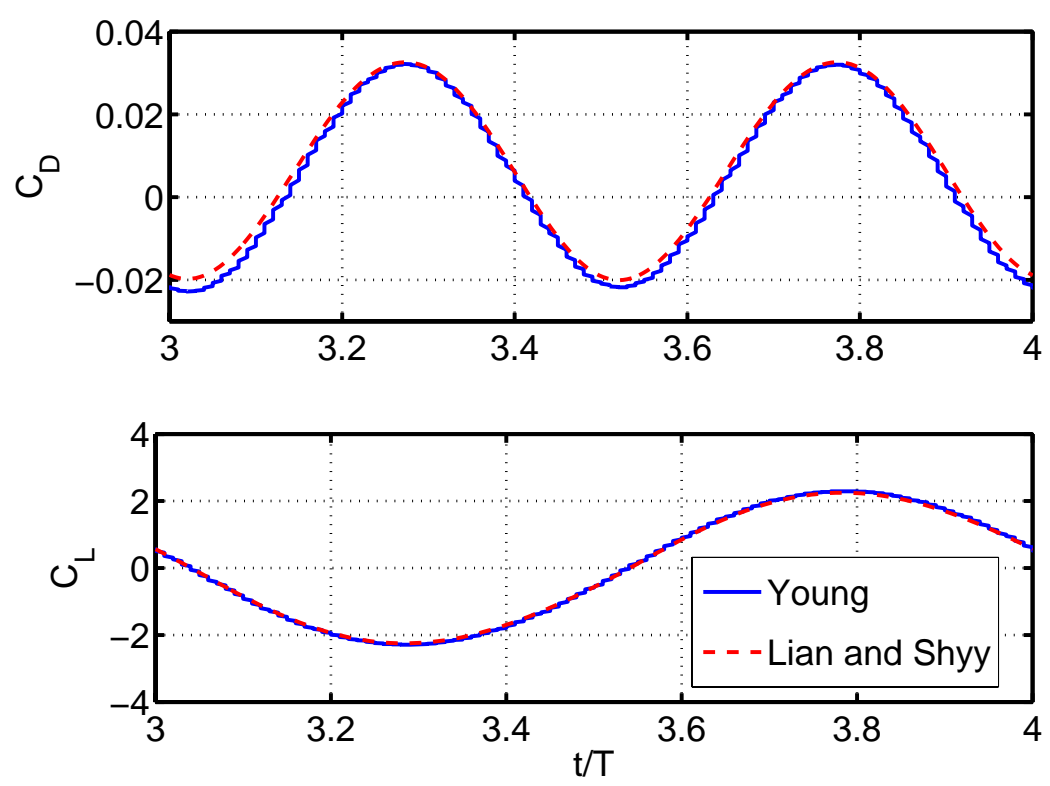

Figure 6. Drag and lift coefficient history at $k=3.93, h_{0}=0.025$, and $S t=0.06$.

As discussed in Eq. (4), the airfoil experiences an instantaneous kinematic angle of attack due to its flapping motion. Intuitively the higher such an angle of attack is, the higher the lift coefficient will be. However, this intuition is based on two assumptions: quasi-steady state and no impact from the wake. Figure 7 clearly demonstrates that the lift variation does not coincide with the kinematic angle of attack, with the lift leading the angle of attack by around $40^{\circ}$. Instead, the lift closely follows the airfoil plunge acceleration, $d^{2} h / d t^{2}$. 
Since the pressure force dominates the total lift, it will be valuable to examine the pressure variation during the airfoil plunging motion. Five representative time instants are chosen, as marked A, B, C, D, and E in Figure 7. The corresponding pressure coefficient distributions are shown in Figure 8. At instant A, the airfoil is in the middle plunging plane $\left(h /\left(h_{0} c\right)=0\right)$ and both the lift and drag coefficients approaches to their minimum magnitudes. There is a cross-over point in the pressure coefficient locating at around $35 \%$ of the chord. After the cross-over point, the pressure on the upper surface is actually larger than the pressure at the corresponding lower surface, which reduces the total lift. During the plunging down motion (instants $\mathrm{B}$ and $\mathrm{C}$ in Figure 8(a)), the cross-over point moves toward the leading edge, which generates negative lift. Once the airfoil starts to plunge upward, the cross-over point moves toward the trailing edge (instants $\mathrm{D}$ and $\mathrm{E}$ in Figure 8(b)). For the studied case, flow history is more important than the kinematic angle of attack in terms of aerodynamic forces, especially the lift. We can draw the same conclusion for other tests with increased plunging amplitude $(0.05 c$ and $0.075 c)$ but same other parameters. These tests suggest that quasi-steady simulation may not be adequate for unsteady simulation, especially when the reduced frequency is considerably high. As we will see soon, in the combined plunging and pitching study with plunging amplitude of $0.75 c$, the kinematic angle of attack seems more responsible for the lift.
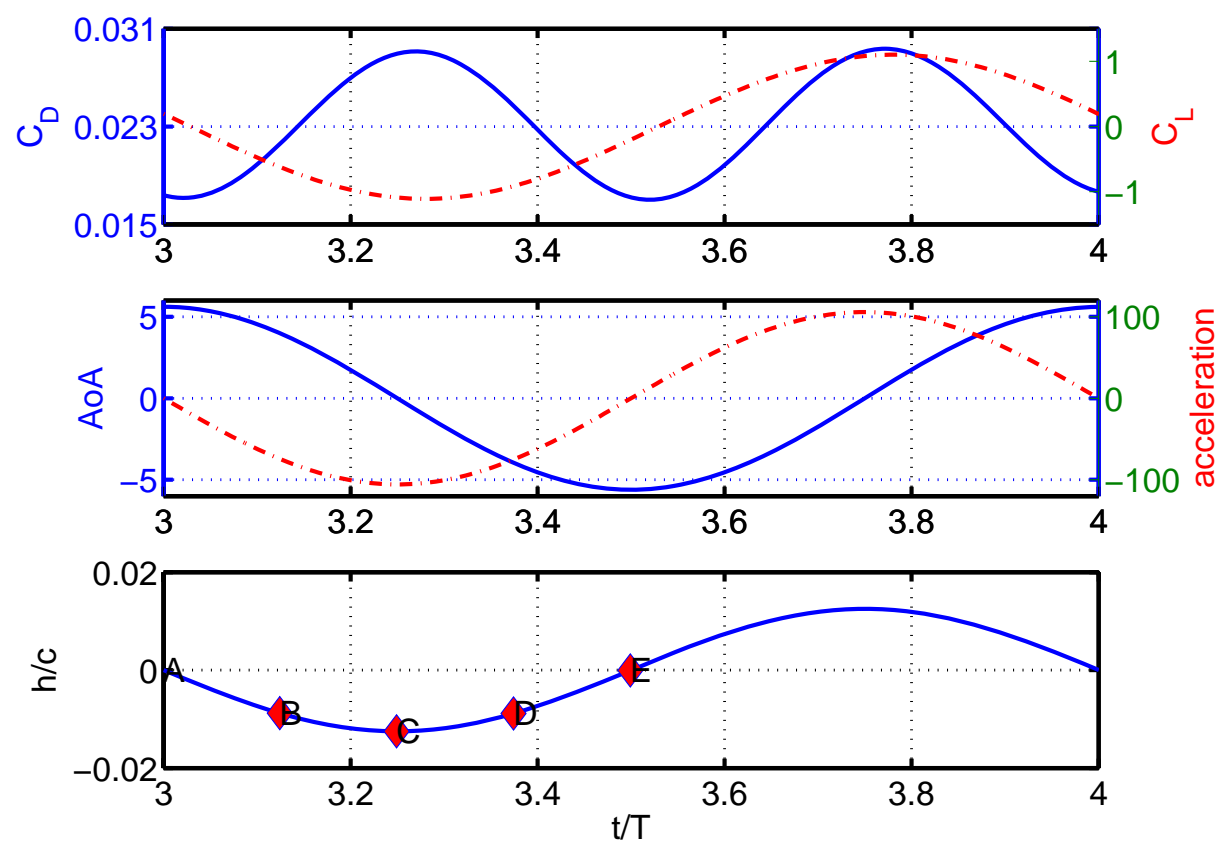

Figure 7. Drag and lift coefficient history at $k=3.93, h_{0}=0.0125$, and $S t=0.03$.

We further inspect the flow structure relative to the airfoil. This is obtained by transforming the velocity field to the coordinate fixed on the plunging airfoil. Figure 9 vividly shows the pattern of the kinematic angle of attack changes from positive to negative, as is consistent with Figure 7. Through the plunge cycle, flow separates near the trailing edge. The separated flow creates a blunt-edged body and vortices are shed alternatively from the trailing edge. A close-up reveals that there are two vortices near the trailing edge at instant A when the airfoil is at its middle plane and starts to plunge down. As shown in Figure 10 (a), one vortex rotates clockwise which is due to the flow separation, and another vortex is small and rotates anticlockwise, indicating it is caused by the airfoil motion. When the airfoil passes the middle plane and moves up, the small vortex rotates clockwise (Figure 10 (b)).

No leading edge vortex is observed when the plunging amplitude is small $(0.0125$ and 0.025$)$. But, it appears with the increase of plunging amplitude. Figure 11 shows the streamlines at the relative coordinate. Leading edge vortex and trailing edge vortex coexist. In the work of Young no leading edge vortex was found at even high amplitude of 0.25 . We gather that the leading edge vortex is caused by the kinematic angle of attack defined in Eq. (4). Hence both large plunge amplitude and frequency will contribute to the appearance of leading edge vortex in similar way. 


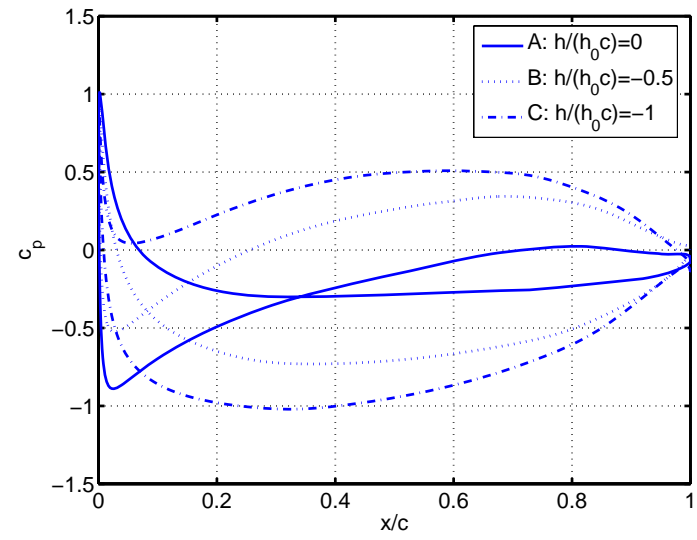

(a)

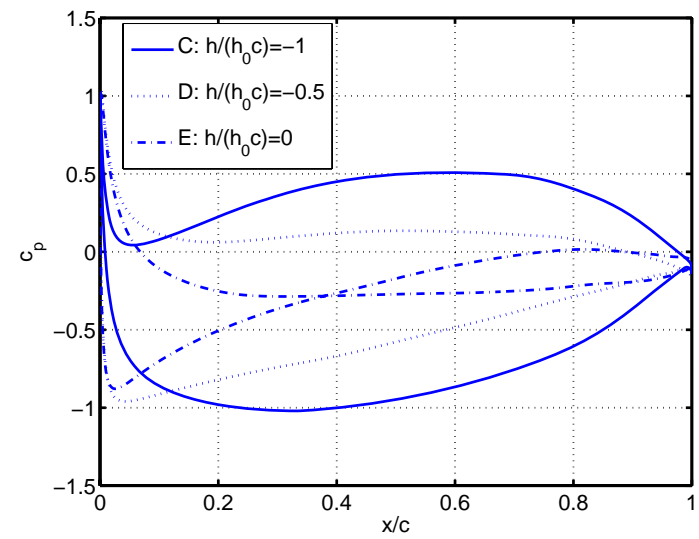

(b)

Figure 8. Pressure coefficient evolvement during half plunging cycle: $k=3.93, h_{0}=0.0125$, and $S t=0.03$. (a) One quarter cycle from instant $\mathrm{A}$ to $\mathrm{C}$ (from middle plane to bottom plane); (b) another quarter cycle from bottom plane to middle plane.

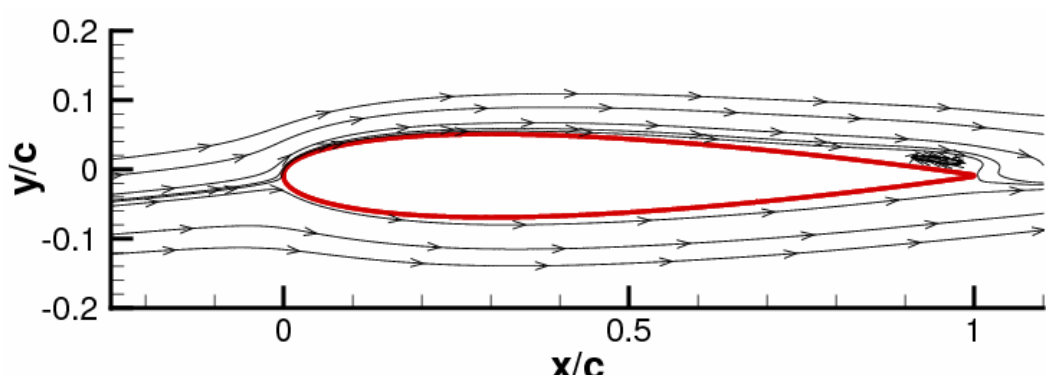

A

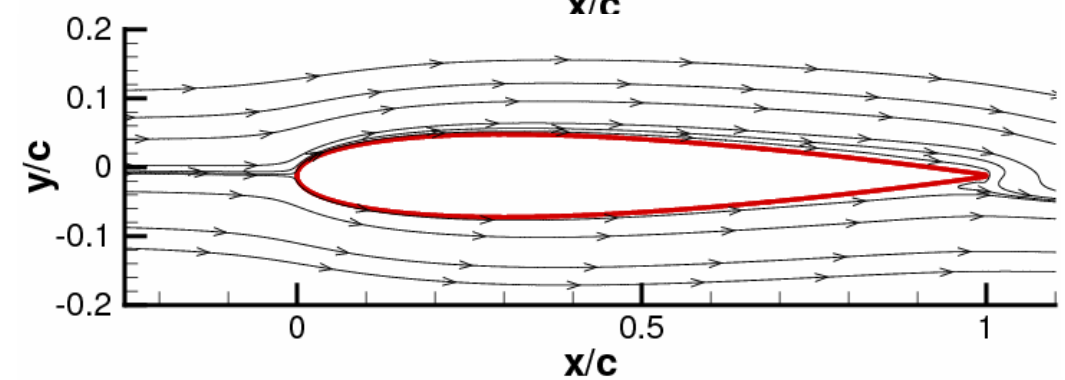

$\mathrm{C}$

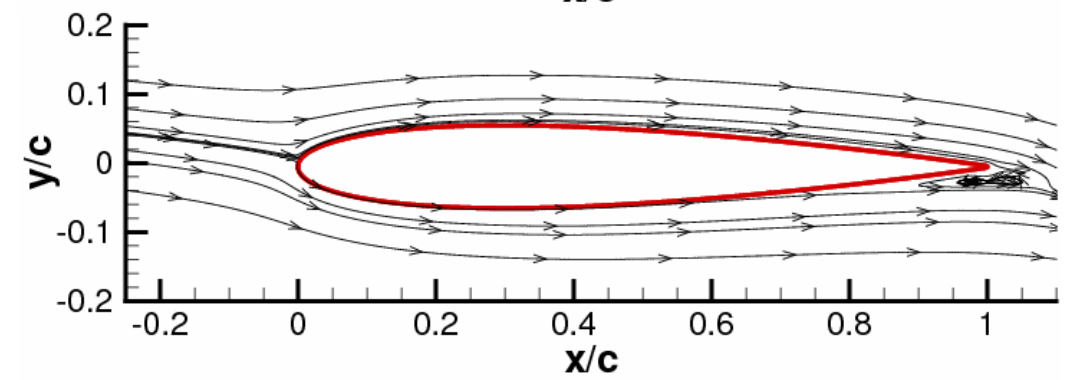

$\mathrm{E}$

Figure 9. Instantaneous streamlines in the relative coordinate during the half flapping cycle. From top to bottom is A through E in Figure 7. $k=3.93, h_{0}=0.0125$, and $S t=0.03$. 


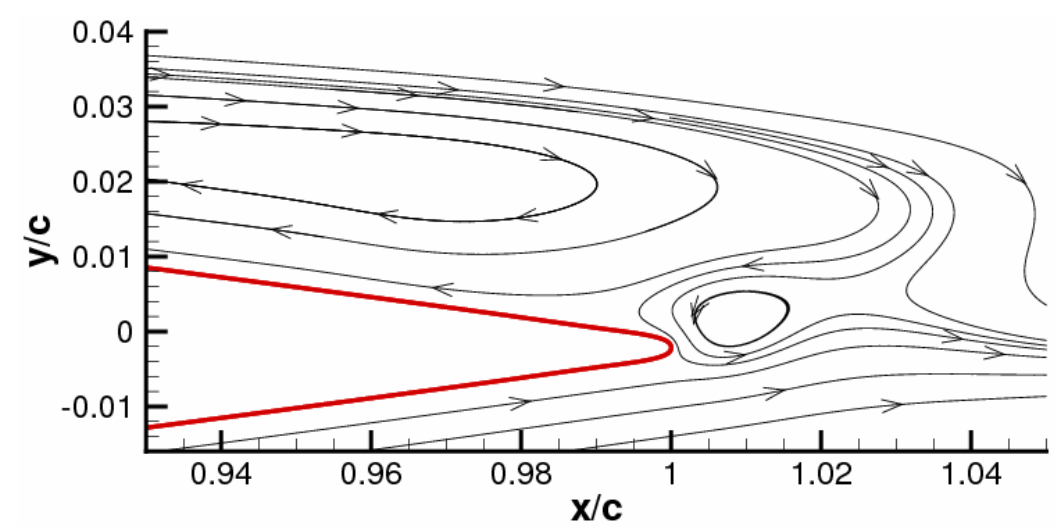

(a)

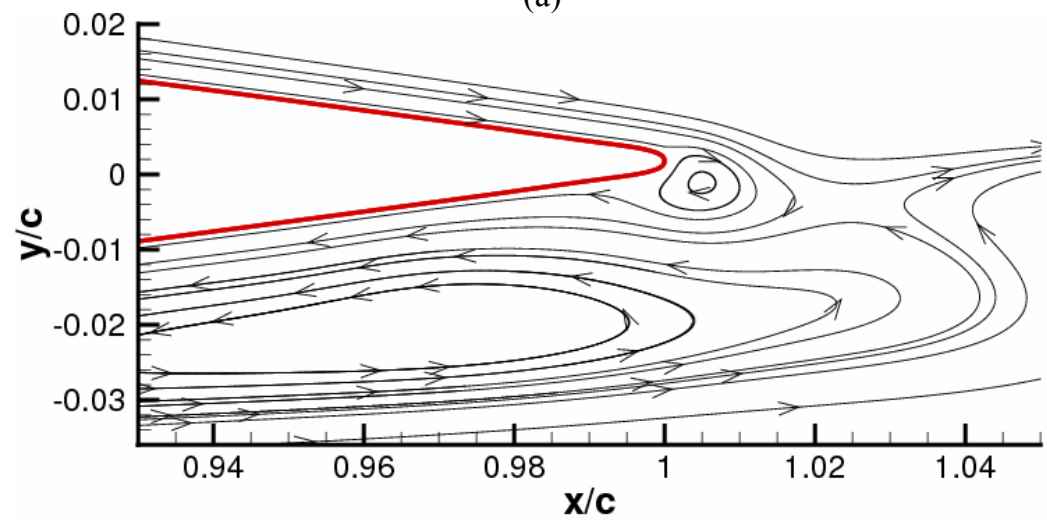

(b)

Figure 10. Close-up of instantaneous streamlines in the relative coordinate during the half flapping cycle. At $k=3.93, h_{0}=0.0125$, and $S t=0.03$. (a) Plunge down at $h /\left(h_{0} c\right)=0$; (b) Plunge up at $h /\left(h_{0} c\right)=0$.

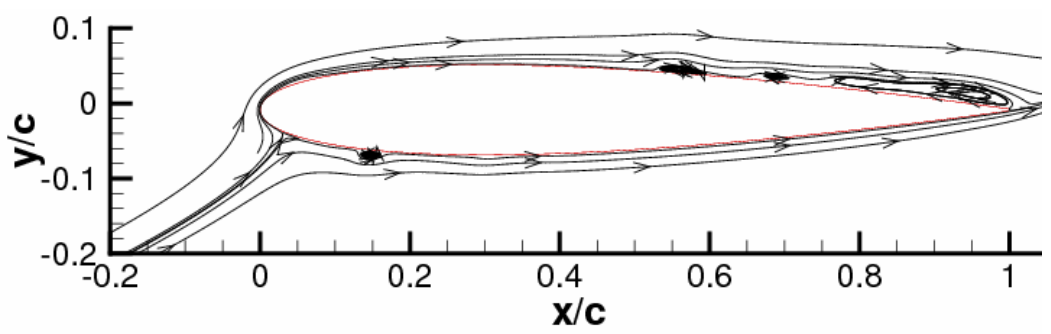

Figure 11. Instantaneous streamlines in the relative coordinate when the airfoil is at the middle plane and plunges down. $k=3.93, h_{0}=0.05$, and $S t=0.125$.

Jones et al. experimentally visualized the wake structures of a plunging airfoil. Depending on the flapping amplitude and frequency, they found the drag-producing Karman street, the neutral wake and the thrustproducing reverse Karman street. They indicated that the wake structure depended on the $k h_{0}(=2 \pi S t)$. However, experiments by Triantafyllou et al. ${ }^{[26]}$, Ohashi and Ishikawa, ${ }^{[35]}$ and Kadlec and Davis ${ }^{[36]}$ showed the wake structure was independently determined by the both $k$ and $h_{0}$, which was further confirmed experimentally ${ }^{[19]}$ and numerically. ${ }^{[9]}$ 
Figure 12 illustrates the streaklines in the wake region at two different flapping amplitudes, both of which show the reverse Karman street and indicate thrust generation. For the considered two cases, flow visualization reveals a clear wake structure near the trailing edge, ${ }^{[19]}$ especially indicating that the diffusivity rate at that region is low. We assume flow is laminar. Simulations by Young showed little difference between laminar and turbulent results. ${ }^{[20]} \mathrm{A}$ large scale similarity with the experimental visualization is apparent.

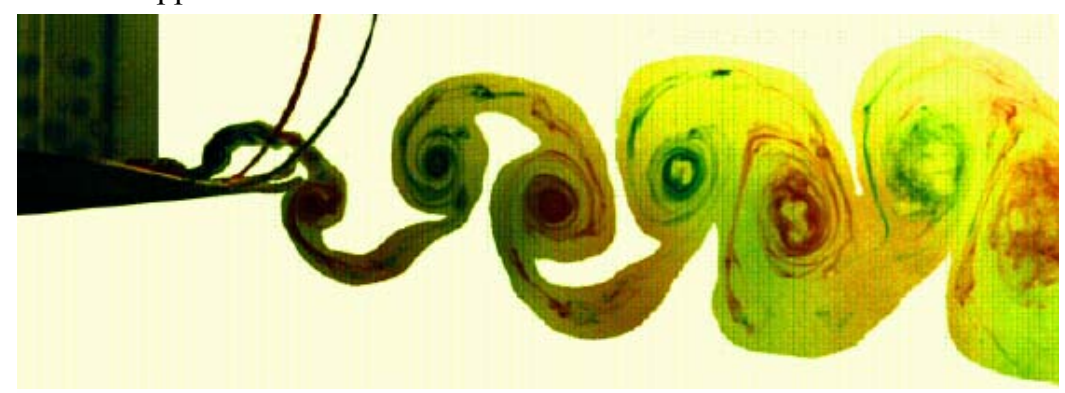

Exp: Lai and Platzer: $k=3.93, h_{0}=0.05$, and $S t=0.125{ }^{[19]}$

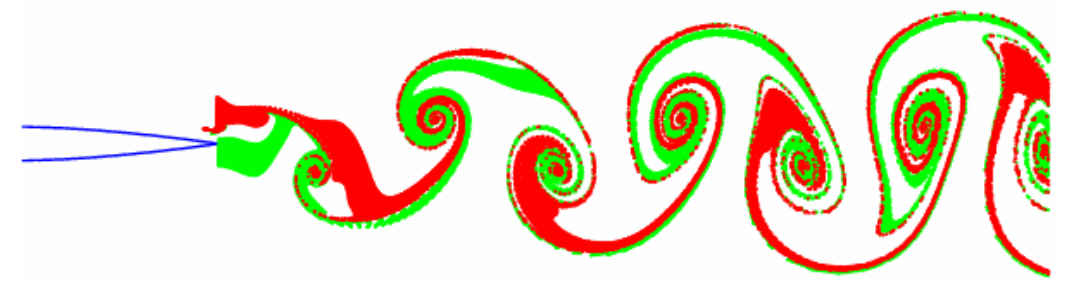

CFD: Lian and Shyy: $k=3.93, h_{0}=0.05$, and $S t=0.125$.

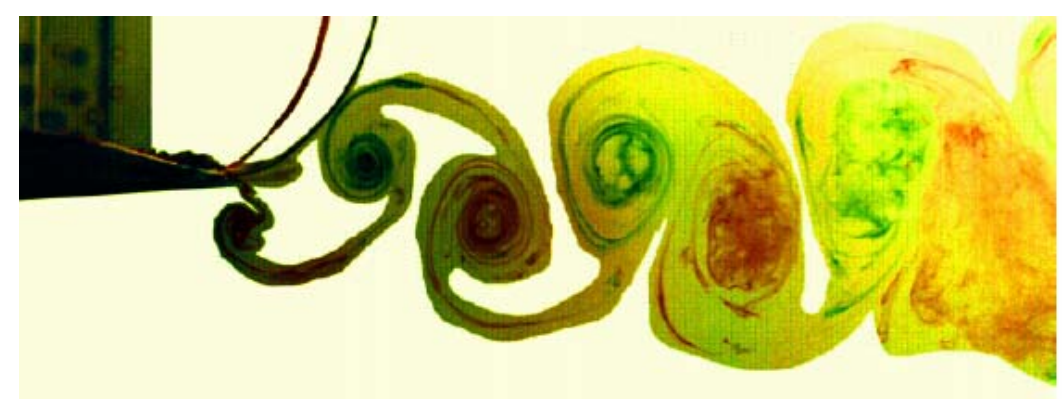

EXP: Lai and Platzer: $k=3.93, h_{0}=0.075$, and $S t=0.188 .{ }^{[19]}$

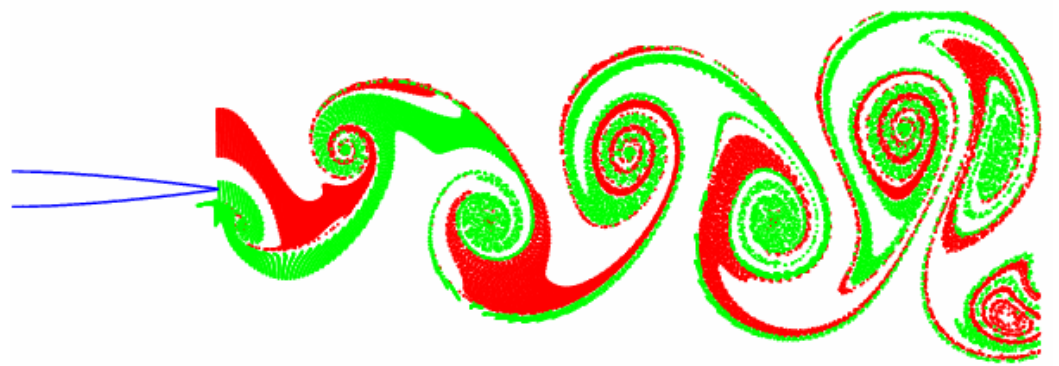

CFD: Lian and Shyy: $k=3.93, h_{0}=0.075$, and $S t=0.188$.

Figure 12. Streakline plot of flow over plunging airfoil NACA0012 at $\mathrm{Re}=20,000$ and reduced frequency of 3.93 .

\section{Combined plunging and pitching motion}

Anderson et al. measured the time-averaged thrust coefficient, input power coefficient, and propulsion efficiency of a NACA0012 airfoil undergoing combined sinusoidal plunging and pitching motion in the testing tank facility at MIT. ${ }^{[7]}$ The tank has the dimensions of $30 \mathrm{~m} \times 2.5 \mathrm{~m} \times 1.2 \mathrm{~m}$ and can run at constant speed up to $3 \mathrm{~m} / \mathrm{s}$. The airfoil has a chord of $10 \mathrm{~cm}$ and span of $60 \mathrm{~cm}$. Circular end plates are fitted to avoid the three-dimensional end effects. A piezoelectric force transducer provides force measurement in three axes while another transducer measures the force transmitted to the chain to find torque. The reported Reynolds number is $\mathrm{Re}=4 \times 10^{4}$, and the pitch axis is located at the $1 / 3$ chord point. 
Among their systematic tests with varied plunging amplitude, nominal angle of attack, and Strouhal number, one of the optimal cases is obtained for the plunging amplitude-to-chord ratio, $h_{0}=0.75$, the phase angle between plunging and pitch (pitch leading plunge) is $75^{\circ}$, and the normal angle of attack, $\alpha_{0}=15^{\circ}$. We study is centered with this setting with varied Strouhal number. The readers should be aware that based on full turbulent simulation with the k- $\omega$ turbulence model, the turbulent Reynolds number in the vicinity of the airfoil is in the order of 100, indicating the Reynolds number of $4 \times 10^{4}$ may not be high enough to prompt full turbulent. On the other hand, laminar flow simulation may not have the resolution to resolve the flow structure at this Reynolds number. We do not claim that we fully resolve the low Reynolds number aerodynamics. But, our main purpose is to look at the aerodynamics based on available analysis tool. For the flapping wing study, we use both laminar and turbulent simulations but the analysis except that shown in Figure 13 is based on laminar flow simulation.

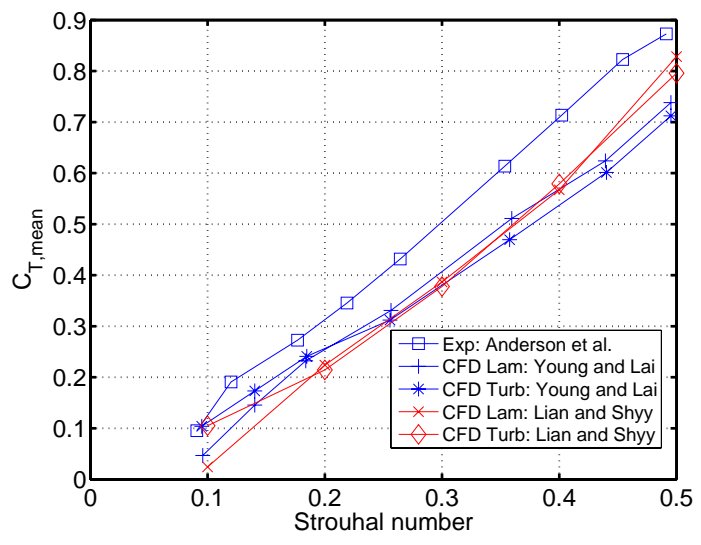

(a)

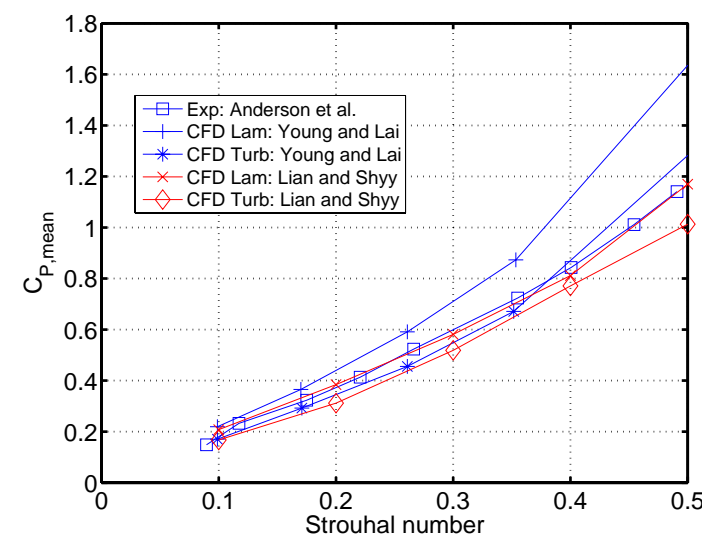

(b)

Figure 13. (a) Thrust coefficient; (b) power efficient as a function of Strouhal number for $h_{0}=0.75, \alpha_{0}=$ $15^{\circ}$, and $\psi=75^{\circ}$.

We compare the mean thrust coefficient and input power at different Strouhal number with both experimental and numerical data in Figure 13. For all the Strouhal number except at Strouhal number of 0.1 , the laminar and turbulent simulations under-predict the mean thrust coefficient. However, the input power coefficients from our laminar flow simulation closely match the measurement by Anderson et al. ${ }^{[7]}$ Overall, all the data shows that the time-averaged thrust and input power increase with the Strouhal number.

Figure 14 shows the time histories of lift, thrust, input power, and moment during one flapping cycle, starting from the middle stroke plane. The Strouhal number is 0.3 and reduced frequency is 0.63 . At the beginning of the cycle, the airfoil plunges down (Figure 15(a)) with the maximum negative pitching angle $\theta$ (Figure 15(b)). However, both the lift and thrust are near their maximum values. The reason, as explained before, is that the airfoil actually experiences a large kinematic angle of attack, $\alpha$ (Figure 15(c)). From Figure 15(d) we know that at instant A the angle of attack due to plunging motion, $\beta$, reaches its maximum. As illustrated in Figure 16 (a), the local flow is deflected. The local flow strikes the airfoil from below instead of in front. The lift, which is perpendicular to the local flow, is then inclined toward upstream, which leads to thrust (Figure 14(b)). Similar conclusions can be drawn at instant B.

When the airfoil reaches its lowest position during the cycle (point $\mathrm{C}$ ), the kinematic angle of attack becomes negative. From Figure 16(c) we also see that the local flow meets the airfoil from above. However, both the lift and thrust coefficients are close to zero. As we mentioned before, the flow history may be responsible for that. After point $\mathrm{C}$, the airfoil starts to reverse its plunging direction. Meanwhile, the leading edge of the airfoil pitches up and the trailing edge pitches down. At instant $\mathrm{D}$, the kinematic angle of attack is negative, and flow is deflected downward. Meanwhile, the lift is tilted towards upstream. Again, the tilted lift leads to thrust. It is a half-cycle from point A to point D, and the other half-cycle from point $\mathrm{E}$ to point $\mathrm{H}$ can be seen as the opposite side of the first half cycle. 


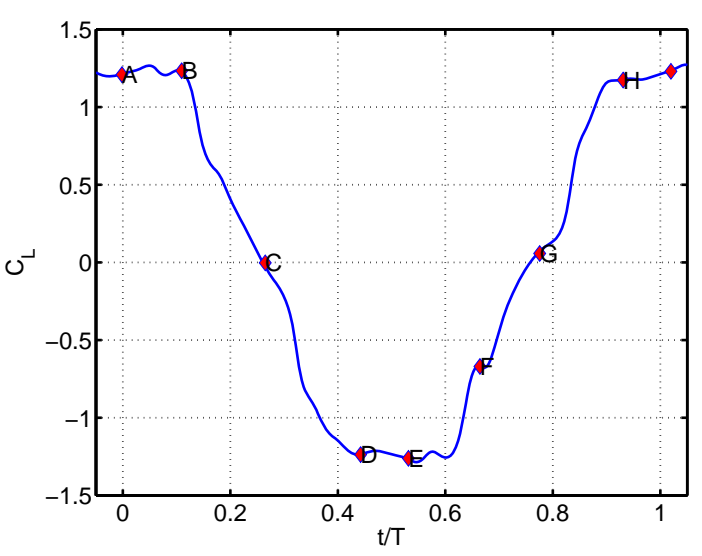

(a)

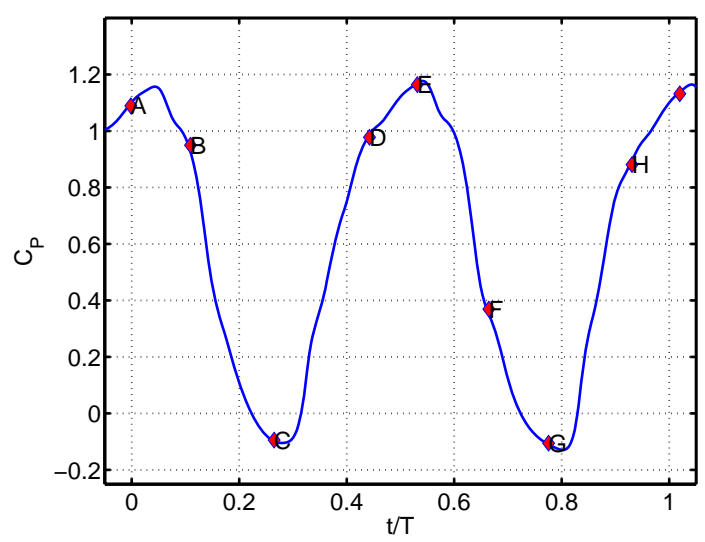

(c)

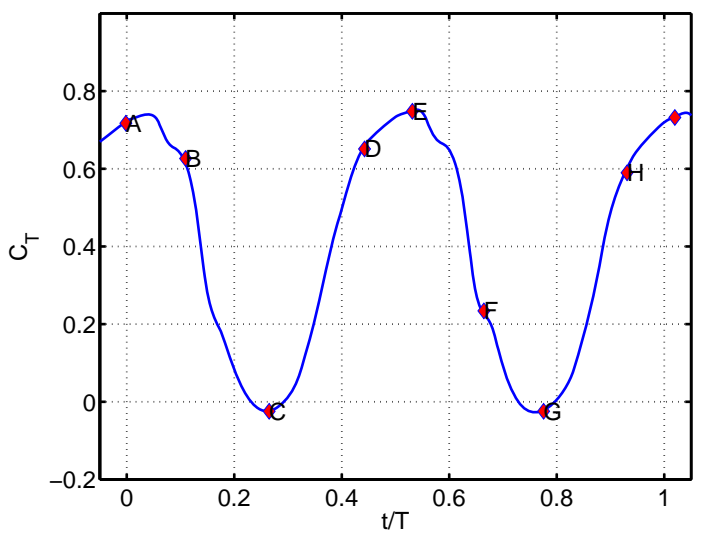

(b)

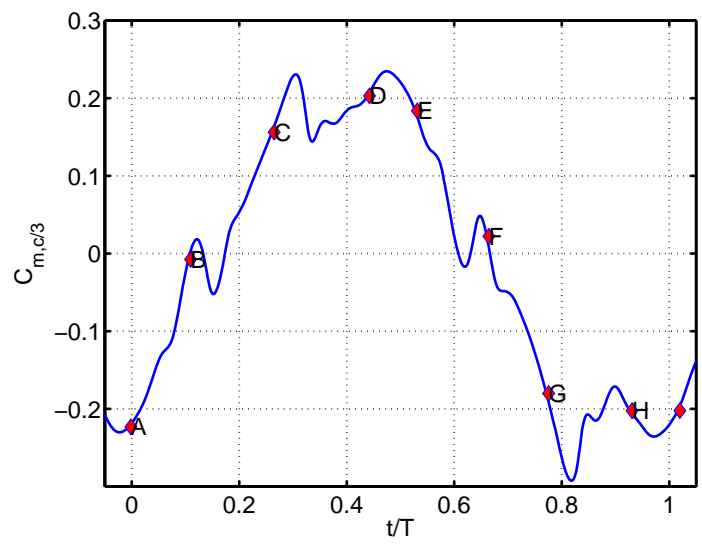

(d)

Figure 14. Time histories of lift, thrust, moment, and input power coefficient for at $h_{0}=0.75, S t=0.3, k=$. $0.63, \psi=75^{\circ}$, and $\alpha_{0}=15^{\circ}$ using laminar simulation.

It is instructive to look at the flow filed to understand how the leading edge vortex enhances the lift and thrust. During the plunging down motion, a leading edge vortex is formed and travels downstream (Figure 16). The vortex core is usually associated with low pressure (Figure 17), which enhances the lift and thrust. When it travels downstream, the vortex gradually loses its coherent structure and strength, and the pressure approaches its ambient value. For example, the pressure within the core at instant $\mathrm{C}$ is about one third of the value at instant A. The vorticity contours at the corresponding time instants are shown in Figure 18. The vortex leaves off the trailing edge and is shed into the wake.

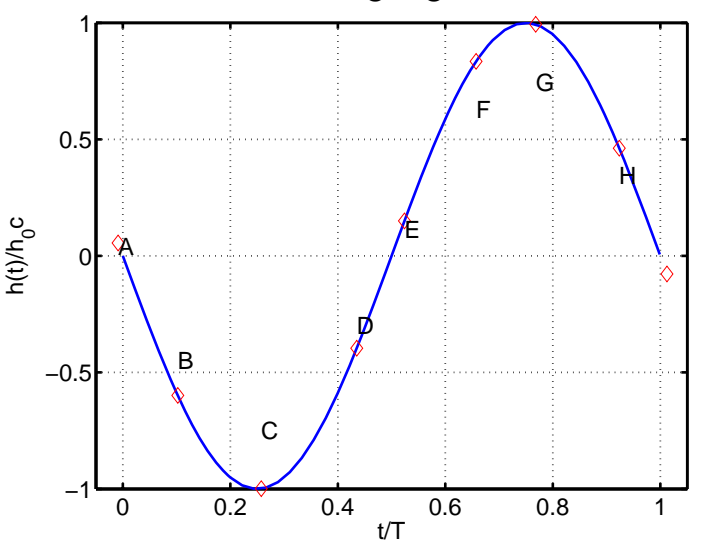

(a)

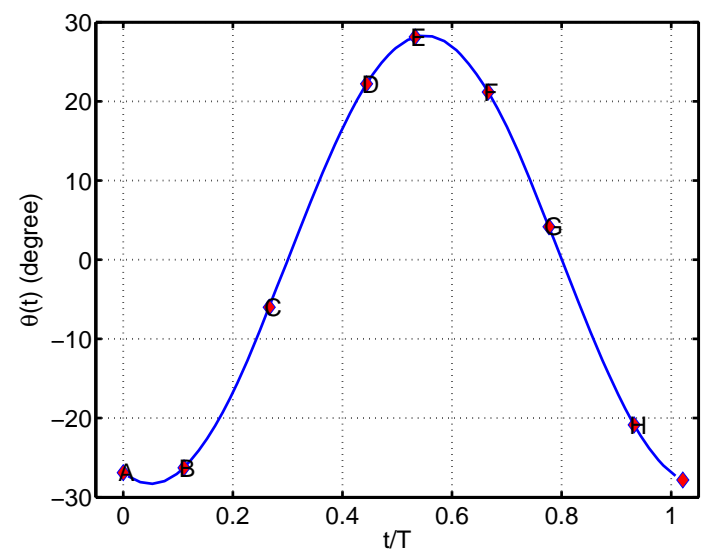

(b) 


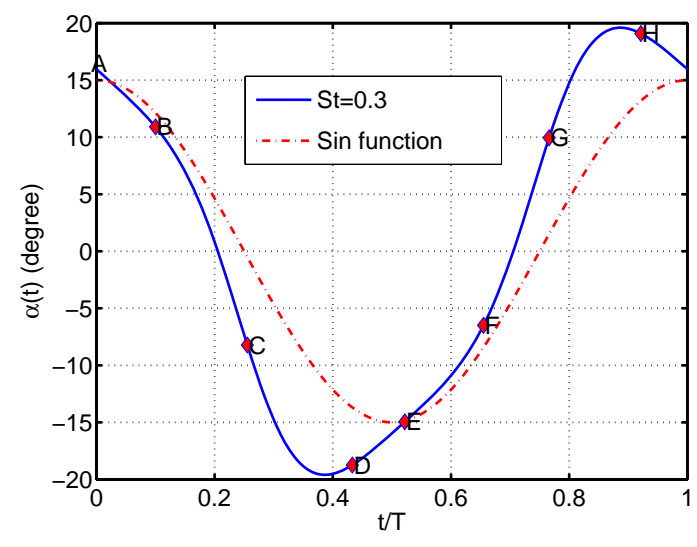

(c)

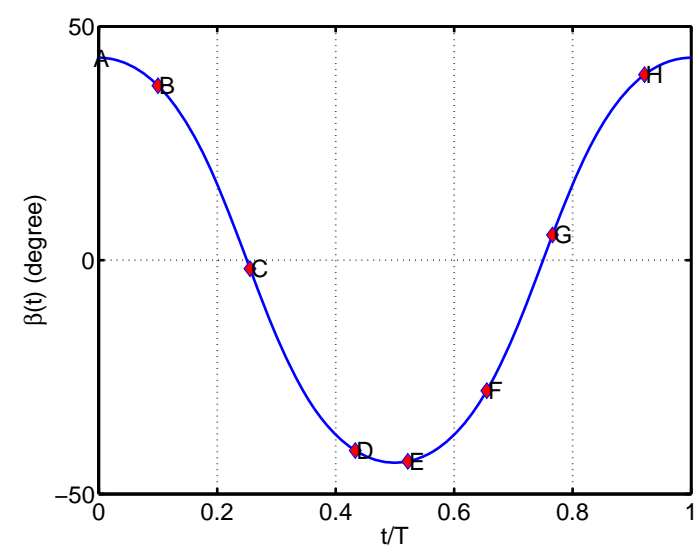

(d)

Figure 15. Time history for at $h_{0}=0.75, S t=0.3, k=0.63, \psi=75^{\circ}$, and $\alpha_{0}=15^{\circ}$.

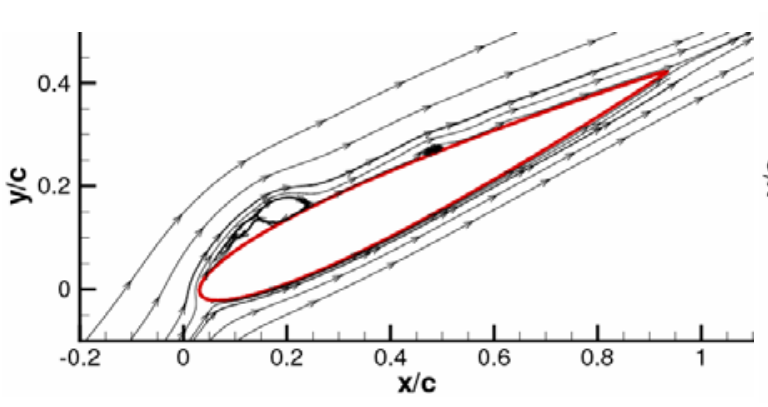

(a)

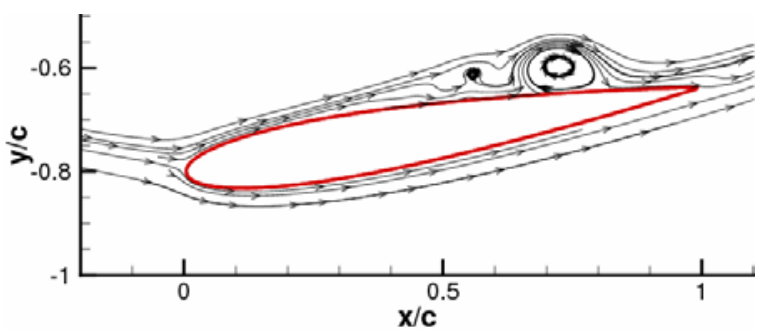

(c)

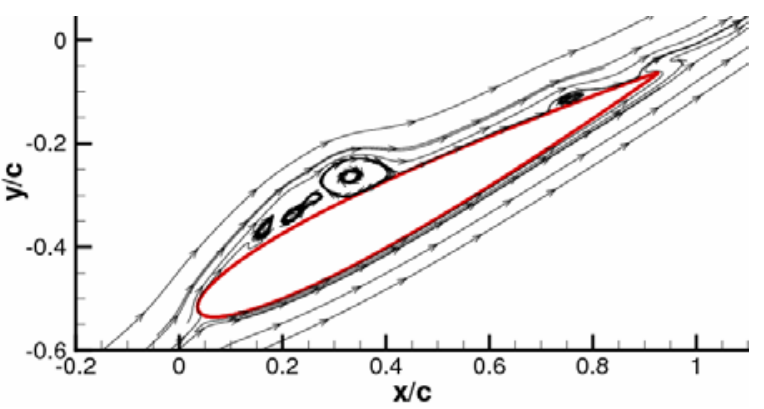

(b)

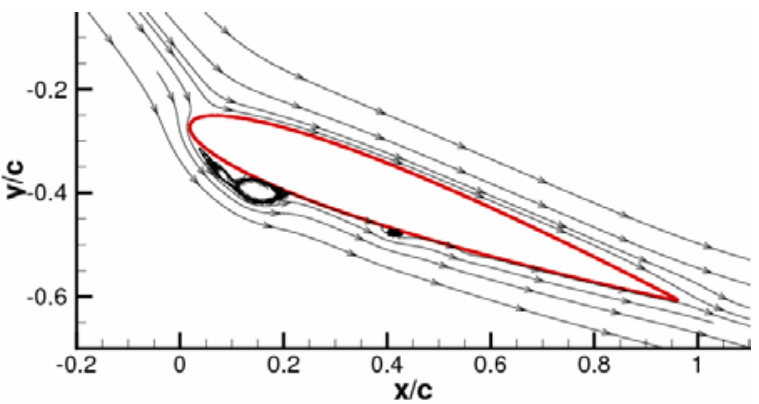

(d)

Figure 16. Streamlines seen from the coordinates fixed on the airfoil. (a) $h /\left(h_{0} c\right)=0$; (b) $h /\left(h_{0} c\right)=-0.6$; (c) $h /\left(h_{0} c\right)=-1 ;$ (d) $h /\left(h_{0} c\right)=-0.4 . h_{0}=0.75, S t=0.3, k=0.63, \psi=75^{\circ}$, and $\alpha_{0}=15^{\circ}$. 


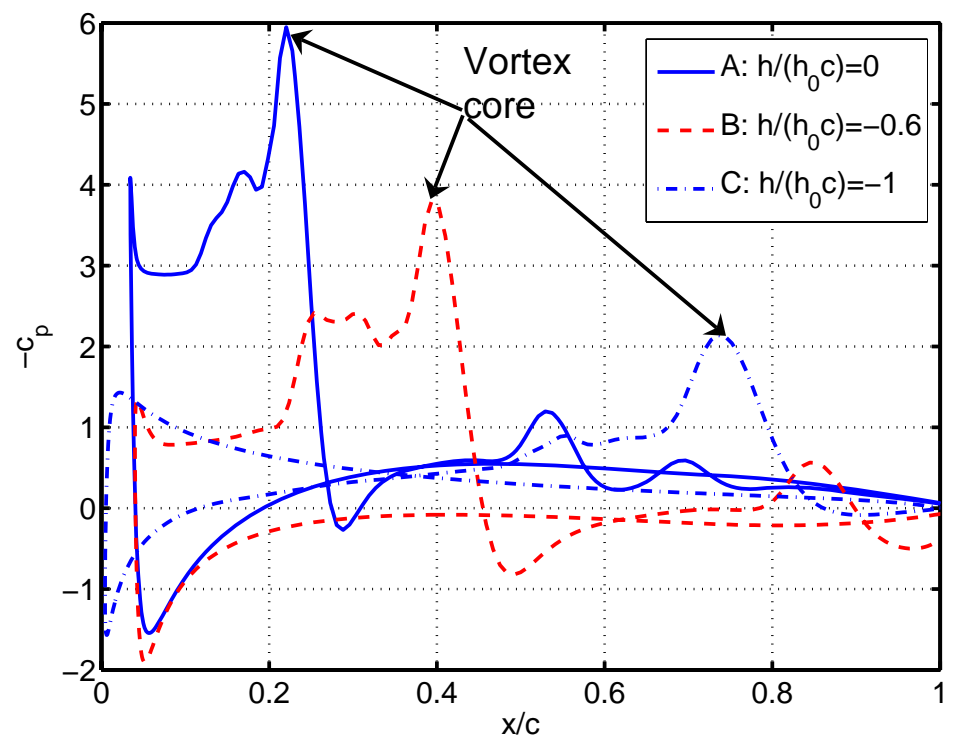

Figure 17. Pressure coefficient distribution at three time instants. $h_{0}=0.75, S t=0.3, k=0.63, \psi=75^{\circ}$, and $\alpha_{0}=15^{\circ}$.

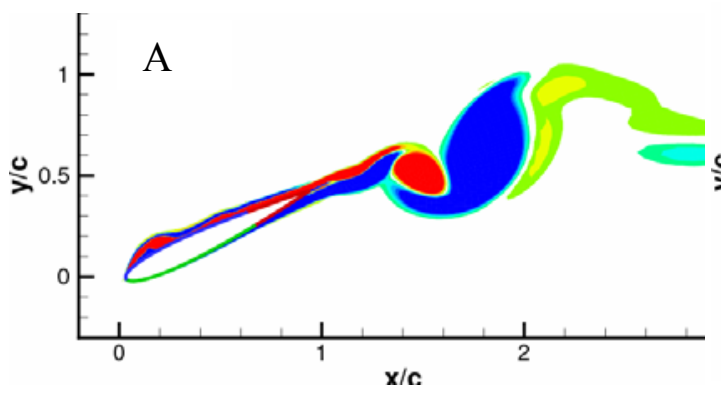

(a)

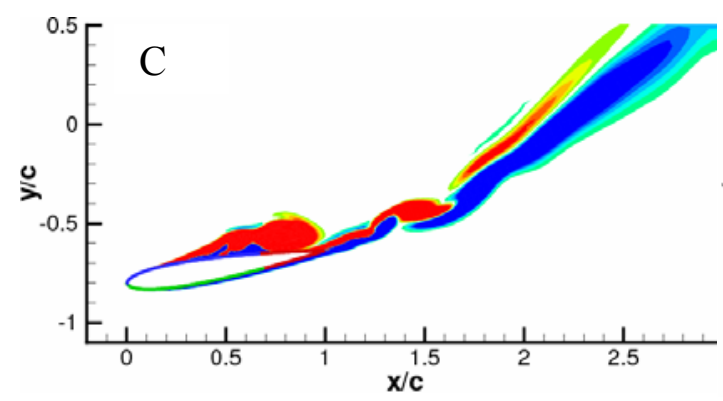

(c)

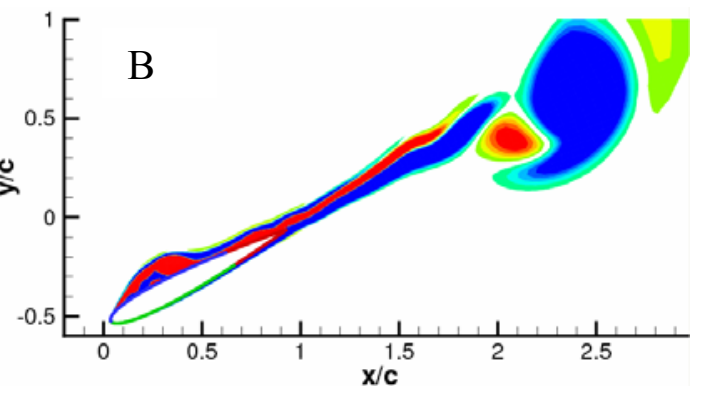

(b)

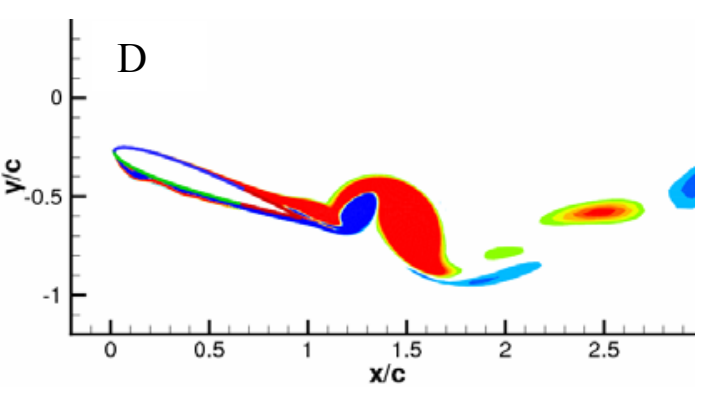

(d)

Figure 18. Vorticity contours at different instants during half flapping cycle. (a) $h /\left(h_{0} c\right)=0$; (b) $h /\left(h_{0} c\right)=-$ 0.6 ; (c) $h /\left(h_{0} c\right)=-1$; (d) $h /\left(h_{0} c\right)=-0.4 . h_{0}=0.75, S t=0.3, k=0.63, \psi=75^{\circ}$, and $\alpha_{0}=15^{\circ}$.

\section{Stationary airfoil in unsteady freestream}

We study the response of a stationary NACA0012 in unsteady flow. The Reynolds number is 40,000 based on the freestream velocity and chord length and the angle of attack is set to $4^{\circ}$. At this Reynolds number and angle of attack, laminar separation bubble causes flow to experience laminar to turbulent 
transition. We use a method by Lian and Shyy ${ }^{[16]}$ to simulate the transitional flow. Figure 19 shows the computed streamlines and the normalized turbulence shear stress $\overline{u^{\prime} v^{\prime}} / U_{\text {ref }}^{2}$. Our simulation predicts that transition occurs at $65 \%$ of the chord based on a relatively low freestream intensity of $0.1 \%$ while $\mathrm{XFoil}^{[32]}$ predicts transition position is $66.7 \%$. The lift and drag coefficients are 0.53 and 0.03 from our simulation and 0.53 and 0.026 from XFoil, respectively.

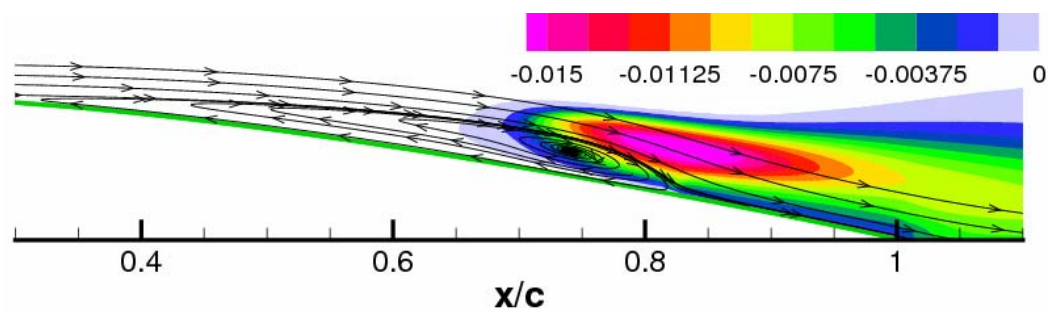

Figure 19. Streamlines and normalized turbulent shear stress over a NACA0012 airfoil for $\alpha=4^{\circ}$.

We limit our focus on head-on gust with a single frequency of $1 \mathrm{~Hz}$ :

$$
U(t)=U_{0}\left(1+N_{A} \sin (\omega t)\right)
$$

it varies sinusoidally with time with a frequency of $\omega$ and variation of $N_{A}$. Here $\omega$ is equal to $2 \pi$ and $N_{A}$ is 0.2 . Figure 20 (a) compare both the lift and drag coefficients. The comparison between the unsteady and quasi-steady is made at the same Reynolds number. Overall, the quasi-steady simulation predicts larger lift and lower drag than the unsteady simulation. The lift curve of the quasi-steady simulation has similar pattern as the unsteady computation, both following the variation of $U^{2} / U_{0}^{2}$. On the other hand, the drag curve from the unsteady simulation is quite different from the quasi-steady prediction.

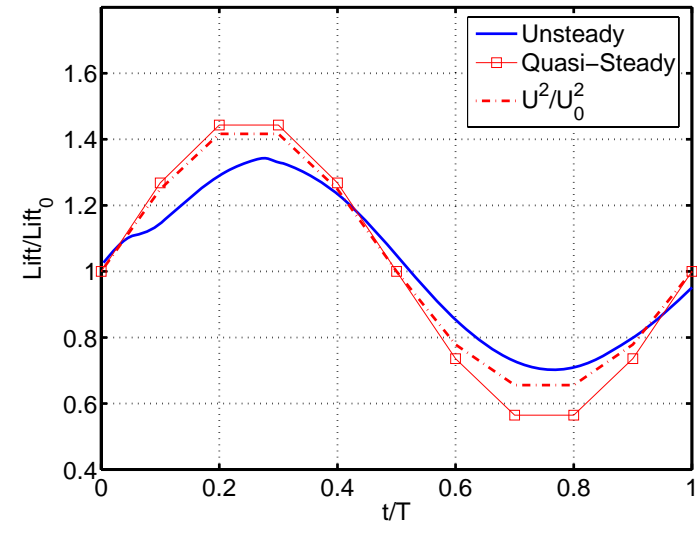

(a)

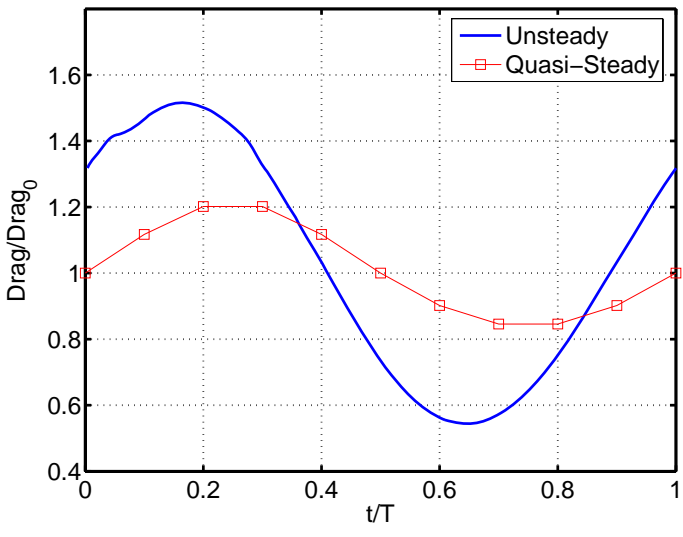

(b)

Figure 20. Comparison of lift and drag coefficients between unsteady and quasi-steady computations of a stationary airfoil. (a): lift coefficient; (b): drag coefficient.

\section{Flapping airfoil in unsteady freestream}

The flapping airfoil follows plunging motion described previously in Eq. (1). The pitching motion is as follows:

$$
\theta(t)=\theta_{0} \cos \left(\omega t+\phi_{\theta}\right)+4^{o}
$$


The other parameters are set to $S t=0.3, k=0.63, h_{0}=0.75, \alpha_{0}=15^{\circ}, \omega=2 \pi$, and $\psi=75^{\circ}$. In the case, the time averaged pitching angle of attack is $4^{\circ}$. The same head-on gust in Eq. (10) is applied to the freestream. Hence, the gust has a frequency of $1 \mathrm{~Hz}$ while the airfoil flapping at $7.4 \mathrm{~Hz}$.

Time histories of the lift and thrust are shown in Figure 21, in which both the lift and thrust are nondimensionalized by their mean values. During each flapping cycle, neither the lift nor the thrust has the symmetric pattern as shown early in Figure 14. We average the lift and thrust in each flapping cycle and plot them in Figure 22. None follows the variation of $U^{2} / U_{0}^{2}$. Instead, the time-averaged thrust decreases as the freestream velocity increases. It seems there is a phase lag between the gust variation and the response from the airfoil.

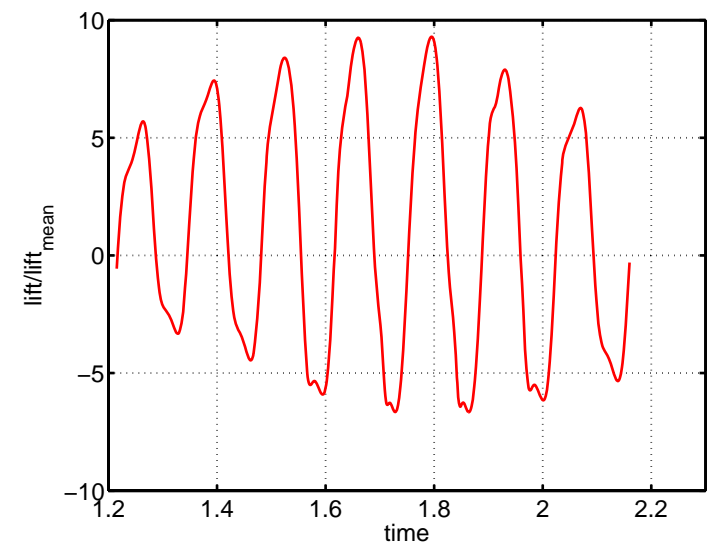

(a)

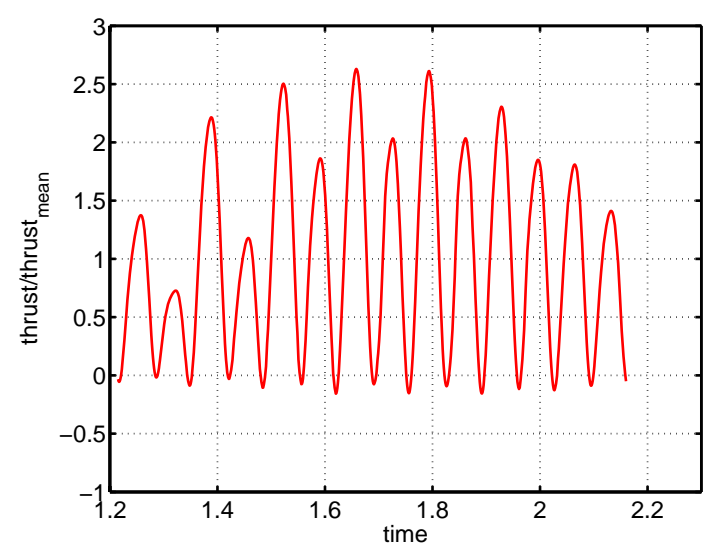

(b)

Figure 21. Force history of a flapping airfoil during one gust cycle. (a) Lift (b) thrust. $h_{0}=0.75, S t=0.3, k$ $=0.63, \psi=75^{\circ}$, and $\alpha_{0}=15^{\circ}$. The ratio between the flapping frequency and gust frequency is 7.4.
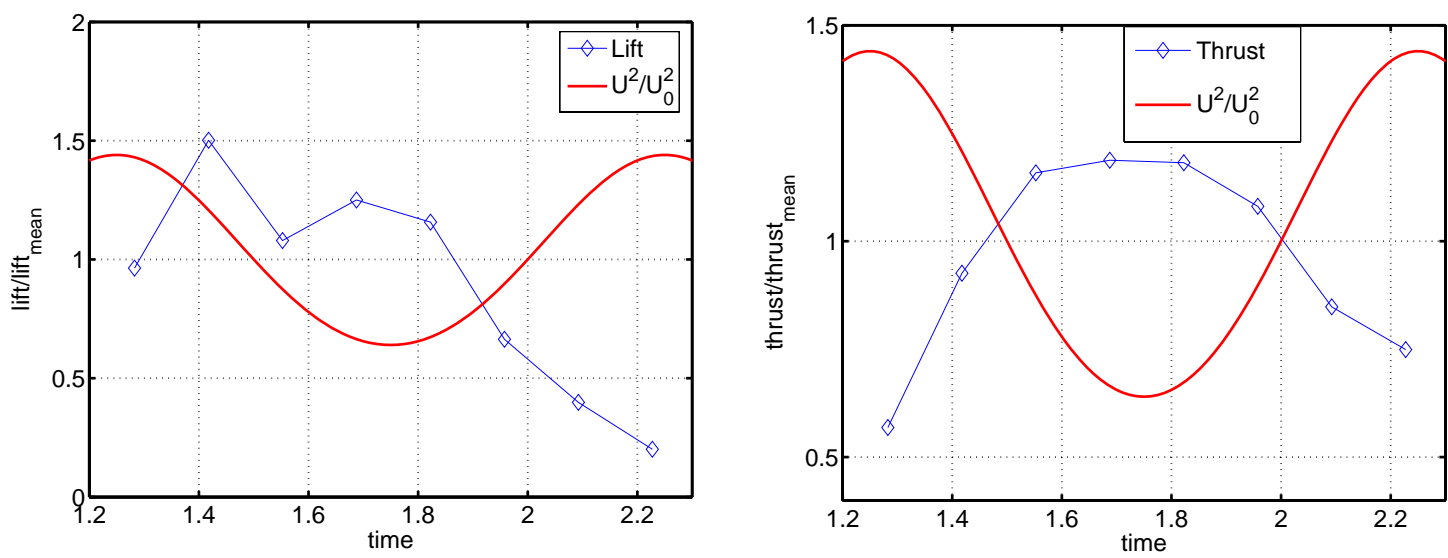

Figure 22. Time-averaged force of a flapping airfoil during one gust cycle. (a) Lift (b) thrust. $h_{0}=0.75, S t=$ $0.3, k=0.63, \psi=75^{\circ}$, and $\alpha_{\mathrm{o}}=15^{\circ}$.

\section{Conclusion}

We use the NACA0012 airfoil as one example to study the flapping airfoil aerodynamics. The Reynolds number is in the range between $1.2 \times 10^{4}$ and $4.0 \times 10^{4}$; the non-dimensional flapping amplitude spans from 0.0125 to 0.75 ; the Strouhal number covers a range between 0.03 and 0.5 . Based on our theoretical analysis and numerical simulation we have the following findings: 
1) Both the reduced frequency and the Strouhal number, characterizing the time scale between flapping and freestream motion and length scales between chord and stroke amplitude, should be considered independently to determine the flapping airfoil aerodynamics, which includes the wake structure, lift and thrust generation, and leading and trailing edge vortex formation.

2) The plunging airfoil can generate thrust or drag depending on the plunging frequency and amplitude. At fixed flapping frequency, increasing the plunging amplitude can make the airfoil from drag generating to thrust generating.

3) When the flapping frequency is fixed, at small the flapping amplitudes $\left(h_{0}\right.$ of 0.0125 and 0.025$)$, there is only trailing edge vortex due to the effective bluff trailing edge and the plunging motion; at large amplitudes $\left(h_{0}\right.$ of 0.05 and 0.075$)$, there are both leading edge vortex and trailing edge vortex.

4) When the reduced frequency is considerably high ( $k$ of 3.93), the time history effect has profound impact, and the lift variation does not follow the change of the kinematic angle of attack.

5) When the plunging amplitude is fixed, increasing the Strouhal number from 0.1 to 0.5 will increase the thrust and input power coefficients.

6) During the flapping, a leading edge vortex enhances the thrust because it generates a low pressure zone. This vortex gradually loses its strength when it travels downstream.

7) The response of a stationary airfoil in gust environment can be approximated with quasi-steady approach when the gust varies with low frequency.

8) The lift and thrust of a flapping airfoil do not follow the change of the gust.

\section{Acknowledgment}

The work presented has been supported in part by the AFOSR. The authors have benefited from the communication with Drs. Joseph Lai and John Young of the Australian Defence Force Academy of Australia.

\section{References}

${ }^{1}$ Grasmeyer, J., and Keenon, M., "Development of the Black Widow Micro Air Vehicle," AIAA Paper 2001-0127, 2001.

${ }^{2}$ Ifju, P., Jenkins, D. A., Ettinger, S., Lian, Y., Shyy, W., and Waszak, M. R., "Flexible Wing Based Micro Air Vehicles," AIAA Paper 2002-0705, 2002.

${ }^{3} \mathrm{http} / / / \mathrm{www}$.proxflyer.com/

${ }^{4}$ Krashanitsa, R., Platanitis, G., Silin, B., and Shkarayev, S., “Aerodynamics and Controls Design for Autonomous Micro Air Vehicles," AIAA paper 2006-6639.

${ }^{5}$ Jones, K. D., Bradshaw, C. J., Papadopoulos, J., and Platzer, M. F., "Development and Flight Testing of Flapping Wing Propelled Micro Air Vehicles," AIAA paper 2003-6549.

${ }^{6}$ Jones, K. D., and Platzer, M. F., “Bio-Inspired Design of FlappingWing Micro Air Vehicles -An Engineer's Perspective," AIAA Paper 2006-0037.

${ }^{7}$ Anderson, J.M., Streitlien, K., Barrett, D.S. \& Triantafyllou, M.S. (1998) Oscillating foils of high propulsive efficiency, J. Fluid Mech., 360, pp. 41-72.

${ }^{8}$ Tuncer, I.H. \& Platzer, M.F. (1996) Thrust generation due to airfoil flapping, Aiaa J., 34, pp. 324-331.

${ }^{9}$ Young, J. \& Lai, J. (2004) Oscillation Frequency and Amplitude Effects on the Wake of a Plunging Airfoil, AIAA Journal, 42(10), pp. 2042-2052.

${ }^{10}$ Triantafyllou, G.S., Triantafyllou, M.S. \& Chryssostomidis, C. (1986) On the Formation of Vortex Streets behind Stationary Cylinders, J. Fluid Mechanics, 170, pp. 461-477.

${ }^{11}$ Zaide, A., and Raveh, D., "Numerical Simulation and Reduced-Order Modeling of Airfoil Gust Response," AIAA Journal, Vol. 44, No. 8, 2006, pp. 1826-1834..

${ }^{12}$ Patil, M. J., and Taylor, D. J., “Gust Response of Highly Flexible Aircraft,” AIAA Paper 2006-1638.

${ }^{13}$ Golubev, V. V., Mankbadi, R. R., Visbal, M. R., Scott, J. R., and Hixon, R., “A Parametric Study of Nonlinear GustAirfoil Interaction,” AIAA Paper 2006-2426.

${ }^{14}$ Yang, G., and Obayashi, S., "Numerical Analyses of Discrete Gust Response for an Aircraft," Journal of Aircraft, Vol. 41, No. 6, 2004, pp. 1353-1359. 
${ }^{15}$ Shyy, W., Jenkins, D.A. \& Smith, R.W. (1997) Study of Adaptive Shape Airfoils at Low Reynolds Number in Oscillatory Flow, AIAA Journal, 35(9), pp. 1545-1548.

${ }^{16}$ Lian, Y., and Shyy, W., "Laminar-Turbulent Transition of a Low Reynolds Number Rigid or Flexible Airfoil," AIAA Paper 2006-3051. Accepted for publication by AIAA Journal.

${ }^{17}$ Koochesfahani, M.M. (1989) Vortical Patterns in the Wake of an Oscillating Airfoil, Aiaa J., 27(9), pp. 1200-1205.

${ }^{18}$ Jones, K.D., Dohring, C.M. \& Platzer, F.M. (1998) Experimental and Computational Investigation of the KnollerBetz Effect, Aiaa J., 36(7), pp. 1240-1246.

${ }^{19}$ Lai, C.S.J. \& Platzer, F.M. (1999) Jet Characteristics of a Plunging Airfoil, Aiaa J., 37(12).

${ }^{20}$ Young, J., Numerical Simulation of the Unsteady Aerodynamics of Flapping Airfoils. Ph.D dissertation, School of Aerospace, Civil and Mechanical Engineering, The University of New South Wales/Australian Defence Force Academy, 2005.

${ }^{21}$ Anderson, J. D. Jr., Introduction to Flight (Third Edition), McGraw-Hill, New York, 1989.

${ }^{22}$ Theodorsen, T., "General Theory of Aerodynamic Instability and Mechanism of Flutter,” NACA-TR-496, 1935.

${ }^{23}$ Garrick, I. E., "Propulsion of a Flapping and Oscillating Airfoil,” NACA Report, No. 567, 1937.

${ }^{24}$ Taylor, K.G., Nudds, L.R. \& Thomas, L.R.A. (2003) Flying and Swimming Annimals Cruise at a Strouhal Number Tuned for High Power Efficiency, Nature, 425(16), pp. 707-711.

${ }^{25}$ Triantafyllou, M.S., Triantafyllou, G.S. \& Gopalkrishnan, R. (1991) Wake Mechanics for Thrust Generation in Oscillating Foils, Physics Fluid A, 12(3), pp. 2835-2837.

${ }^{26}$ Triantafyllou, G.S., Triantafyllou, M.S., and Grosenbaugh, M. A., "Optimal Thrust Development in Oscillating Foils with Application to Fish Propulsion,” Journal of Fluids and Structures, Vol. 7, 1993, pp. 205-224.

${ }^{27}$ Bandyopadhyay, P.R., Castano, J.M., Nedderman, W.H. \& Donnelly, M.J. (2000) Experimental Simulation of FishInspired Unsteady Vortex Dynamics on a Rigid Cylinder, ASME Journal of Fluids Engineering, 122(2), pp. 219-238.

${ }^{28}$ Triantafyllou, M. S., Triantafyllow, G. S., and Yue, D. K. P., "Hydrodynamics of Fishlike Swimming," Annual Review of Fluid Mechanics, Vol. 32, 2000, pp. 33-53.

${ }^{29}$ Patankar, S. V., and Spalding, D. B., "A Calculation Procedure for Heat, Mass and Momentum Transfer in Three-Dimensional Parabolic Flows,” Int. J. Heat Mass Transf., Vol. 15, 1972, pp. 1787-1806.

${ }^{30}$ Shyy, W. Computational Modeling for Fluid Flow and Interfacial Transport, 1997, New York, Elsevier.

${ }^{31}$ Wilcox, D., Turbulence Modeling for CFD (Second Edition), DCW Industries, Inc, La Canada, California, 2000.

${ }^{32}$ Drela, M., and Giles, M. B., "Viscous-Inviscid Analysis of Transonic and Low Reynolds Number Airfoils," AIAA Journal, Vol. 23, No. 10, pp. 1347-1355, 1987.

${ }^{33}$ Tuncer, H.I. \& Platzer, F.M. (2000) Computational Study of Flapping Airfoil Aerodynamics, Journal of Aircarft, 37(3), pp. 514-520.

${ }^{34}$ Young, J., personal communication, 2006.

${ }^{35}$ Ohashi, H., and Ishikawa, N., "Visualization Study of a Flow Near the Trailing Edge of an Oscillating Airfoil," Bulletin of the Japanese Society of Mechanical Engineers, Vol. 15, 1972, pp. 840-845.

${ }^{36}$ Kadlec, R. A., and Davis, S. S., "Visualization of Quasi-periodic Flows," AIAA Journal, Vol. 17, 1979, $1164-1169$. 